\title{
Crystallography and Riemann Surfaces
}

\author{
V. Elser \\ Department of Physics, Cornell University, \\ Ithaca, NY 14853, USA \\ ve10@cornell.edu
}

\begin{abstract}
The level set of an elliptic function is a doubly periodic point set in $\mathbb{C}$. To obtain a wider spectrum of point sets, we consider, more generally, a Riemann surface $\mathcal{S}$ immersed in $\mathbb{C}^{2}$ and its sections ("cuts") by $\mathbb{C}$. More specifically, we consider surfaces $\mathcal{S}$ defined in terms of a fundamental surface element obtained as a conformal map of triangular domains in $\mathbb{C}$. The discrete group of isometries of $\mathbb{C}^{2}$ generated by reflections in the triangle edges leaves $\mathcal{S}$ invariant and generalizes double-periodicity. Our main result concerns the special case of maps of right triangles, with the right angle being a regular point of the map. For this class of maps we show that only seven Riemann surfaces, when cut, form point sets that are discrete in $\mathbb{C}$. Their isometry groups all have a rank 4 lattice subgroup, but only three of the corresponding point sets are doubly periodic in $\mathbb{C}$. The remaining surfaces form quasiperiodic point sets closely related to the vertex sets of quasiperiodic tilings. In fact, vertex sets of familiar tilings are recovered in all cases by applying the construction to a piecewise flat approximation of the corresponding Riemann surface. The geometry of point sets formed by cuts of Riemann surfaces is no less "rigid" than the geometry determined by a tiling, and has the distinct advantage in having a regular behavior with respect to the complex parameter which specifies the cut.
\end{abstract}

\section{Introduction}

Crystallography is concerned with point sets in $\mathbb{R}^{n}$ that are discrete and distributed more or less uniformly. In "classical" crystallography, periodicity was imposed as well, but this restriction is not considered as fundamental in the "modern" era. With the discovery of intermetallic quasicrystals [1] in the early 1980s, it became clear that there exist aperiodic point sets that share a basic property with periodic point sets. This property should really be associated with a distribution: in this case, the distribution formed by placing a Dirac delta at each point of the set. In these terms, the class of aperiodic sets singled out by crystallography is characterized by the property that the Fourier transform of the corresponding distribution has support on a lattice [2]. The rank of this "Fourier 
lattice" equals the dimension of space for periodic sets, and exceeds it (but is still finite), in the case of quasiperiodic sets. The vertex set of the Penrose tiling of the plane [3] is a familiar example of a quasiperiodic set.

The standard construction of quasiperiodic sets $\mathcal{A}$ begins by embedding $\mathbb{R}^{n}=Y$ in a larger Euclidean space, $\mathbb{R}^{m+n}=X \times Y$. Into $X \times Y$ one then immerses a smooth $m$-manifold $\mathcal{S}$ that is (i) transversal to $Y$, and (ii) invariant under the action of a lattice $\Lambda \subset X \times Y$ generated by $n+m$ linearly independent translations in $X \times Y$. Point sets $\mathcal{A} \subset Y$ are obtained as sections ("cuts") of $\mathcal{S}$ by spaces parallel to $Y$. More formally, in terms of the standard projections

$$
\begin{aligned}
& \pi_{X}: \mathcal{S} \rightarrow X, \\
& \pi_{Y}: \mathcal{S} \rightarrow Y,
\end{aligned}
$$

the section of $\mathcal{S}$ at $x \in X$ is the set-valued map

$$
\mathcal{A}(x)=\pi_{Y} \circ \pi_{X}^{-1}(x)
$$

Periodicity or quasiperiodicity of $\mathcal{A}(x)$ is determined by the rank of the lattice $\Lambda_{Y}=$ $\Lambda \cap Y$. Since the generators of $\Lambda$ were assumed to be linearly independent, $\operatorname{rk}\left(\Lambda_{Y}\right) \leq n$. Quasiperiodicity corresponds to $\mathrm{rk}\left(\Lambda_{Y}\right)<n$, with complete absence of periodicity characterized by $\Lambda_{Y}=\{0\}$. An important motivation for constructing quasiperiodic sets, in this context, is the fact that symmetry groups that cannot be realized by periodic point sets in $\mathbb{R}^{n}$, can be realized by periodic surfaces in $\mathbb{R}^{m+n}$.

Transversality and periodicity are relatively mild restrictions on the manifold $\mathcal{S}$, called the "atomic surface" by physicists. A further restriction, one which leads to point sets called "model sets" [4], is to require that $\mathcal{S}$ is the $\Lambda$-orbit of a polytope in $X$. The algorithm which constructs $\mathcal{A}(x)$ from such $\mathcal{S}$ naturally leads to the terminology "window" or "acceptance domain" for the corresponding polytopes. Model sets can always be organized into finitely many tile shapes, and, because of this simplicity, have dominated the study of quasiperiodic sets.

A different viewpoint on the construction of $\mathcal{S}$, pioneered by Kalugin [5] and Katz [6], emphasizes the continuity properties of $\mathcal{A}(x)$ with respect to $x$. Consider in more detail the construction of a model set: $\mathcal{S}=\mathcal{P} \times\{0\}+\Lambda$, where $\mathcal{P} \subset X$ is a polytope. Now, if $x \in \mathcal{P}+\pi_{X}(\lambda)$ for some $\lambda \in \Lambda$, then $y=\pi_{Y}(\lambda) \in \mathcal{A}(x)$. However, now consider what happens when $x$ crosses the boundary of $\mathcal{P}+\pi_{X}(\lambda)$. As $x$ "falls off the edge of the earth", the corresponding point $y$ in the point set $\mathcal{A}(x)$ disappears. By the same process, of course, points can spontaneously appear "out of thin air". To gain control over these processes, Kalugin [5] and Katz [6] advocated a restriction on $\mathcal{P}$, in relation to $\Lambda$, such that whenever $x$ falls off the edge of one polytope, $\mathcal{P}+\pi_{X}(\lambda)$, it falls within another, say $\mathcal{P}+\pi_{X}\left(\lambda^{\prime}\right)$. This restriction corresponds mathematically to the statement that the boundaries of the disconnected components of $\mathcal{S}=\mathcal{P} \times\{0\}+\Lambda$ can be "glued" together to form a topological manifold without boundary.

In the process of restoring transversality to the glued complex of polytopes one encounters the problems addressed by singularity theory. The map $\pi_{X}$ should now be a smooth (but not necessarily one-to-one) map of $m$-mainfolds. In the trivial situation, when $\pi_{X}$ has no singularities, $\mathcal{S}$ must be diffeomorphic to a collection of hyperplanes. This is the situation explored by Levitov [7] for point sets in two and three dimensions and various symmetry groups. 
When $m=n=2$ and $\mathcal{S}$ is a generic 2-manifold, we have the classic result of Whitney [8] that the stable singularities of smooth maps, such as $\pi_{X}$, are folds and cusps, having respectively codimension one and two. Because the cusp is always accompanied by two folds, the locus of singular values of $\pi_{X}$ consists of curves. The space $X$ is thus populated by singular curves such that whenever $x$ crosses a curve, a pair of points in $\mathcal{A}(x)$ merge and annihilate. One motivation for the present work was the desire to eliminate this point-merging singularity to the greatest extent possible.

By giving the 2-manifold $\mathcal{S}$ a complex structure, and identifying $X$ with the complex plane, we impose additional regularity by insisting that $\pi_{X}$ is locally holomorphic. The singularities of $\pi_{X}$ will then be isolated points. A construction that naturally leads to a $\pi_{X}$ with this property is to let $\mathcal{S}$ be (locally) the graph of a holomorphic function, $f: X \rightarrow Y$. Globally this corresponds to a Riemann surface $\mathcal{S}$ immersed in $\mathbb{C}^{2}=X \times Y$ and having an atlas of compatible charts in $X$. The other ingredient needed by our construction is some way to guarantee that $\mathcal{S}$ is invariant with respect to a lattice $\Lambda$. We meet this challenge by using conformal maps between triangles to define a fundamental graph of $\mathcal{S}$. Schwarz reflections in the triangle edges extend this graph and generate the isometry group of $\mathcal{S}$. For appropriate choices of triangles, the isometry group has a lattice subgroup with the desired properties.

In the second half of this paper we classify a subset of all Riemann surfaces generated by conformal maps of triangles. This subset is characterized by the property that the conformal map is regular at one vertex of the triangles and that the edges at this vertex make the largest possible angle, $\pi / 2$. With the only other restriction being that the corresponding point sets $\mathcal{A}(x)$ are discrete in $Y$, one arrives at a set of seven surfaces. Four of these are quasiperiodic. The point set obtained from a section of one of them is shown in Fig. 1. Also shown in Fig. 1 is a much studied model set [9]: a tiling of boats, stars, and jesters' caps (whose vertices coincide with a subset of the Penrose-tiling vertex set). The point set determined by the Riemann surface can be said to be approximated by the vertex set of the tiling by a systematic process that renders the Riemann surface piecewise flat. The other surfaces obtained in our partial classification, when flattened, also produce familiar tilings (Fig. 3).

\section{Riemann Surfaces Generated by Conformal Maps}

\subsection{Immersed Riemann Surfaces}

We consider Riemann surfaces as analytically continued holomorphic functions, interpreted geometrically as surfaces immersed in $\mathbb{C}^{2}$. Our treatment follows closely the notation and terminology of Ahlfors [10].

Definition 2.1. A function element $F=(U, f)$ consists of a domain $U \subset \mathbb{C}$ and a holomorphic function $f: U \rightarrow \mathbb{C}$.

Definition 2.2. Function elements $F_{1}=\left(U_{1}, f_{1}\right)$ and $F_{2}=\left(U_{2}, f_{2}\right)$ are direct analytic continuations of each other iff $V=U_{1} \cap U_{2} \neq \emptyset$ and $f_{1}=f_{2}$ when restricted to $V$. 


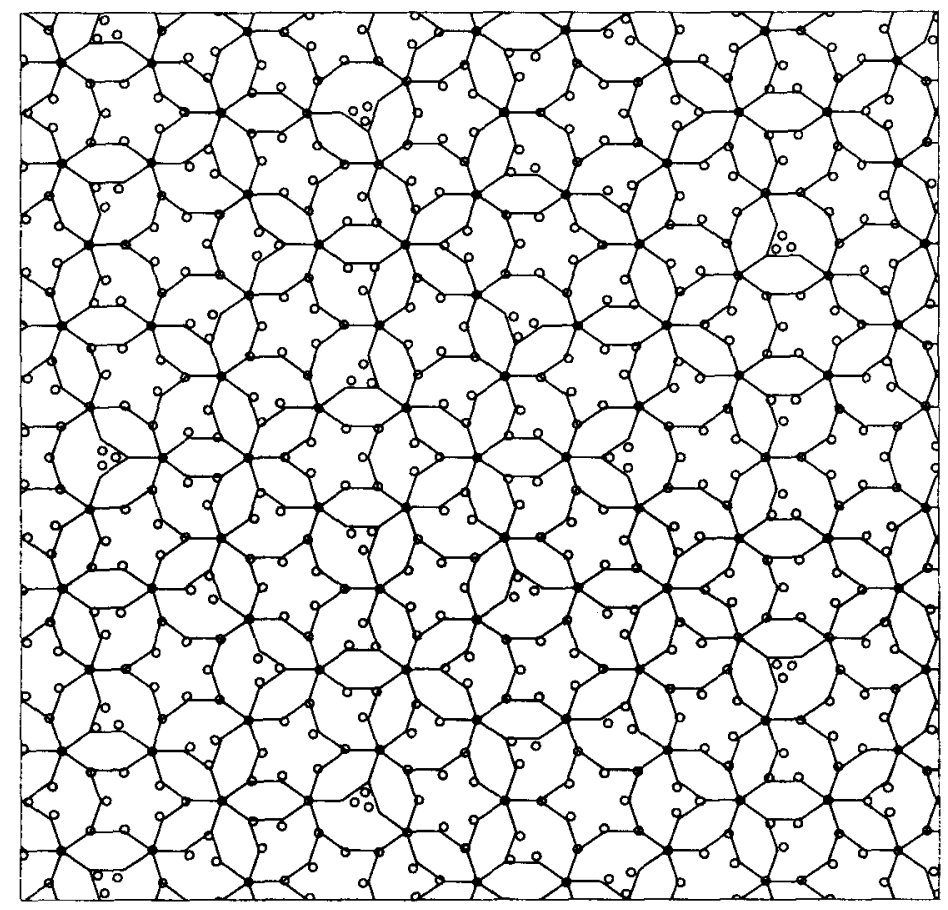

Fig. 1. Point set (small circles) given by the section of a Riemann surface compared with a tiling of boats, stars, and jesters' caps [9].

Definition 2.3. The complete, global analytic function determined by function element $F_{0}=\left(U_{0}, f_{0}\right)$ is the maximal collection of function elements $\mathcal{F}$ such that for any $F_{i} \in \mathcal{F}$ there exists a chain of function elements $F_{0}, \ldots, F_{i}$, all in $\mathcal{F}$, with every link in the chain a direct analytic continuation.

Up to this point the set of function elements comprising a complete, global analytic function $\mathcal{F}$ only possesses the discrete topology, where $F_{1} \cap F_{2}=\emptyset$ whenever $F_{1}$ and $F_{2}$ are distinct elements of $\mathcal{F}$. By refining this topology we can identify $\mathcal{F}$ with a surface and, ultimately, a Riemann surface. Consider a pair of function elements in $\mathcal{F}, F_{1}=\left(U_{1}, f_{1}\right)$ and $F_{2}=\left(U_{2}, f_{2}\right)$. In the refined topology we define the intersection by

$$
F_{1} \cap F_{2}= \begin{cases}F_{3}=\left(U_{3}, f_{3}\right) & \text { if } F_{1} \text { and } F_{2} \text { are related by direct analytic } \\ \text { continuation, } & \text { otherwise, }\end{cases}
$$

where $U_{3}=U_{1} \cap U_{2}$ and $f_{3}$ is $f_{1}=f_{2}$ restricted to $U_{3}$. It is straightforward to check that this defines a valid topology. Moreover, the projection $\pi: \mathcal{F} \rightarrow \mathbb{C}$ given by

$$
\pi:(U, f) \mapsto U,
$$

provides the complex charts that identify $\mathcal{F}$ with a Riemann surface. 
Throughout the rest of this paper we are mostly interested in Riemann surfaces immersed in $\mathbb{C}^{2}$.

Definition 2.4. Let $\mathcal{F}$ be a complete, global analytic function. The immersed Riemann surface $\mathcal{S}$ corresponding to $\mathcal{F}$ is the image of the immersion $\Psi: \mathcal{F} \rightarrow \mathbb{C}^{2}$ given by

$$
\Psi:(U, f) \mapsto\{(x, f(x)): x \in U\} .
$$

Notation. We denote the first component of $\mathbb{C}^{2}$ by $X$, the second by $Y$.

If we restrict the immersion $\Psi$ to a single function element, $F_{0}=\left(U_{0}, f_{0}\right)$, we obtain the graph

$$
\mathcal{S}_{0}=\left\{\left(x, f_{0}(x)\right) \in X \times Y: x \in U_{0}\right\} .
$$

Thus $\mathcal{S}_{0}$ represents a piece of $\mathcal{S}$ and in fact determines all of $\mathcal{S} ; \mathcal{S}$ is connected because every pair of function elements in a complete global analytic function is related by a chain of direct analytic continuations. $\mathcal{S}$ is the completion of $\mathcal{S}_{0}$.

Notation. We write $\left[\mathcal{S}_{0}\right]$ to denote the completion of the graph $\mathcal{S}_{0}$.

Since all subsequent references to "Riemann surface" will be as a surface immersed in $X \times Y$, we drop the qualifier "immersed" below. We also omit the term "complete", since the only instances of incomplete surfaces, graphs, will always be identified as such. Given a Riemann surface $\mathcal{S}$, we frequently make use of the projections

$$
\begin{aligned}
& \pi_{X}: \mathcal{S} \rightarrow X, \\
& \pi_{Y}: \mathcal{S} \rightarrow Y .
\end{aligned}
$$

The historical construction of Riemann surfaces we have followed can be criticized for its inequivalent treatment of the spaces $X$ and $Y$. We can correct this fault by insisting that the functions $f$ appearing in the function elements $(U, f)$ are not just holomorphic in their respective domains $U$, but conformal (holomorphic with holomorphic inverse). The graph (7) could then be equally written as

$$
\mathcal{S}_{0}=\left\{\left(f_{0}^{-1}(y), y\right) \in X \times Y: y \in V_{0}\right\},
$$

where $V_{0}=f_{0}\left(U_{0}\right)$. If this "inversion", or interchange of $X$ with $Y$, is to work for all function elements $(U, f)$, then one must remove all points $x_{0} \in U$, where $f$ behaves locally as $f(x)-f\left(x_{0}\right)=c\left(x-x_{0}\right)^{m}+\cdots$, with $m>1$. These correspond to branch points of the map $\pi_{Y}$. Conversely, had we begun with the inverted function elements our Riemann surface would have included branch points of $\pi_{X}$, i.e. points where $f$ is singular. In keeping with tradition we augment our definition of a Riemann surface $\mathcal{S}$ to include all points $\left(x_{0}, y_{0}\right)$ where $\mathcal{S}$ behaves locally like the algebraic curve $\left(y-y_{0}\right)^{n}=c\left(x-x_{0}\right)^{m}$, where $m$ and $n$ are positive integers.

Definition 2.5. A point $(x, y) \in \mathcal{S}$ is regular if the corresponding complete, global analytic function contains a function element $(U, f)$, with $x \in U$ and $f$ conformal at $x$. A point which is not regular is singular. 


\subsection{Transformations}

Two transformations of Riemann surfaces are needed in our discussion of symmetry properties. These are defined in terms of their action on the spaces $X$ and $Y$ and induce a transformation on Riemann surfaces as subsets of $X \times Y$. Let $(x, y)$ be a general point in $X \times Y$ and define the following transformations:

$$
\begin{aligned}
\tau(a, b ; c, d):(x, y) & \mapsto(a x+b, c y+d), \\
\sigma:(x, y) & \mapsto(\bar{x}, \bar{y}) .
\end{aligned}
$$

Transformation $\tau$ (for complex constants $a, b, c$, and $d$ ) is the general bilinear map while $\sigma$ corresponds to Schwarz reflection (componentwise complex conjugation).

Lemma 2.1. If $\mathcal{S}$ is a Riemann surface and $T$ is either of the transformations $\tau$ or $\sigma$, then $T \mathcal{S}$ is again a Riemann surface.

Proof. Write $T(x, y)=\left(T_{X} x, T_{Y} y\right)$ where $T_{X}$ and $T_{Y}$ are just maps of the complex plane. Since $\mathcal{S}$ corresponds to a complete global analytic function $\mathcal{F}$, we need to verify that $T \mathcal{S}$ corresponds to some other complete global analytic function $\mathcal{F}_{T}$. From our definitions we see that $\mathcal{F}_{T}$ is obtained from $\mathcal{F}$ by substituting each function element $F=(U, f) \in \mathcal{F}$ by $F_{T}=\left(T_{X} U, T_{Y} \circ f \circ T_{X}^{-1}\right)$. It is easily checked that $T_{X}$ is open and $T_{Y} \circ f \circ T_{X}^{-1}$ is holomorphic for both of the transformations being considered. Thus $F_{T}$ remains a valid function element. One also verifies that the direct analytic continuation relationships among function elements are unchanged by these transformations.

Corollary 2.2. If $\mathcal{S}_{0}$ is a graph and $T$ is either of the transformations $\tau$ or $\sigma$, then $\left[T \mathcal{S}_{0}\right]=T\left[\mathcal{S}_{0}\right]$.

Two special transformations are rotations and translations, for which we introduce the following notation:

$$
\begin{aligned}
r(\theta, \varphi) & =\tau\left(e^{i \theta}, 0 ; e^{i \varphi}, 0\right) \\
t(u, v) & =\tau(0, u ; 0, v) .
\end{aligned}
$$

More generally, transformations $T: X \times Y \rightarrow X \times Y$ which act isometrically on the spaces $X$ and $Y$ are just the products of Euclidean motions in $X$ and $Y$. Isometries of Euclidean spaces normally include reflections; to preserve the structure of the immersed Riemann surface, however, any reflection in $X$ (complex conjugation) must be accompanied by a reflection in $Y$.

Definition 2.6. The isometries of $X \times Y$ is the group of transformations generated by $\sigma, r(\theta, \varphi)$, and $t(u, v)$.

In what follows we use the term "isometry" only in this sense. Isometries which preserve a Riemann surface $\mathcal{S}$ are called isometries of $\mathcal{S}$ and form a group. The maximal group of isometries is called the isometry group of $\mathcal{S}$. 
Definition 2.7. The group of proper isometries of $X \times Y$ is the normal subgroup of isometries generated by $r(\theta, \varphi)$ and $t(u, v)$. Any element of the coset, $\sigma g$, where $g$ is a proper isometry, is called a Schwarz reflection.

\subsection{Surfaces Generated by Conformal Maps of Triangles}

We now focus on the class of Riemann surfaces determined by graphs which solve a purely geometrical problem: the conformal map between two bounded and open triangular regions, ${ }^{1} P \subset X$ and $Q \subset Y$. The Riemann mapping theorem [10] asserts there exists a conformal map $f: P \rightarrow Q$ that extends to a homeomorphism of the closures $\bar{P}$ and $\bar{Q}$. By fixing the relative ordering of the vertices of $\bar{P}$ and $\bar{Q}$, the homeomorphism, and hence $f$, is determined uniquely. This defines the graph

$$
P \mid Q=\{(x, f(x)): x \in P\},
$$

and a corresponding Riemann surface $[P \mid Q]$. The closure of $P \mid Q$ is defined analogously and is written $\bar{P} \mid \bar{Q}$. One of the main benefits of using a conformal map of triangles to determine a Riemann surface $\mathcal{S}$ is that its isometry group can be understood simply in terms of its action on a partition of $\mathcal{S}$ into tiles.

Just as $\bar{P}$ can be decomposed into an interior $P$, edges which bound $P$, and vertices which bound each edge, there is a corresponding cell decomposition of the graph $\bar{P} \mid \bar{Q}$. For example, if $P_{1}$ is one vertex of $\bar{P}$, and $f\left(P_{1}\right)=Q_{1}$ is its image in $\bar{Q}$, then we use the symbol $P_{1} \mid Q_{1}$ to represent the corresponding vertex of $\bar{P} \mid \bar{Q}$. Each vertex of $\bar{P} \mid \bar{Q}$ is associated with two angles, a vertex angle of $P$ and the corresponding vertex angle of $Q$. Let the three angle pairs be $\alpha_{i}, \beta_{i}, i=1,2,3$. If $\alpha_{i}=\beta_{i}$ for all $i$, then $P$ is similar to $Q$ and $f$ is just a linear map. Because the corresponding Riemann surface would be trivial (a plane) we exclude this case. It is impossible to have $\alpha_{i} \neq \beta_{i}$ for just one $i$ since then the angle sum could not be $\pi$ in both triangles. Thus we must have at least two vertices with unequal angles. At these vertices $f$ fails to be conformal. Any vertex of $\bar{P} \mid \bar{Q}$ where the corresponding angles in $P$ and $Q$ are unequal is called a singular vertex. The singular vertices of $\bar{P} \mid \bar{Q}$ are the only singular points of $\bar{P} \mid \bar{Q}$.

The edges of $\bar{P} \mid \bar{Q}$ (associated with each pair of vertices $i j=12,13,23$ ) are effectively the generators of the isometry group of $[P \mid Q]$. By $P_{12} \mid Q_{12}$ we mean the graph given by the restriction of $f$ to the edge $P_{12}$ of $\bar{P}$ with image an edge $Q_{12}$ of $\bar{Q}$. Consider the triangles $P^{\prime}$ and $Q^{\prime}$ obtained from $P$ and $Q$ by reflection in these edges. The graph $P^{\prime} \mid Q^{\prime}$, determined by the conformal map $g: P^{\prime} \rightarrow Q^{\prime}$, is clearly related to $P \mid Q$ by an isometry of $X \times Y$, a Schwarz reflection which we call $\sigma_{12}$. Because $\sigma_{12}$ fixes every point of $P_{12} \mid Q_{12}$, we have that $f(x)=g(x)$ for all $x \in P_{12}$. A basic result from complex analysis then tells us that the function elements $(P, f)$ and $\left(P^{\prime}, g\right)$ are related by analytic continuation. Thus $[P \mid Q]=\left[P^{\prime} \mid Q^{\prime}\right]=\left[\sigma_{12}(P \mid Q)\right]=\sigma_{12}[P \mid Q]$, by Corollary 2.2 .

Definition 2.8. The group $G$ generated by the Schwarz reflections $\sigma_{i j}$ which fix the three edges of a triangular graph $P \mid Q$ is called the edge group of $P \mid Q$. The edge group of $P \mid Q$ is a subgroup of the isometry group of $[P \mid Q]$.

\footnotetext{
${ }^{1}$ The case where either $P$ or $Q$ is a half-plane leads to the familiar theory of doubly periodic functions.
} 
In order to show that the edge group of a triangular graph is the maximal isometry group, we first need to refine the sets on which these groups act.

Notation. The symbol $\breve{S}$ corresponds to the Riemann surface $\mathcal{S}$ whose singular points have been removed.

Definition 2.9. A real curve of the Riemann surface $\mathcal{S}$ is any curve $\Gamma \subset \check{\mathcal{S}}$, homeomorphic to $\mathbb{R}$, and pointwise invariant with respect to a Schwarz reflection.

Since both $\pi_{X}: \check{\mathcal{S}} \rightarrow X$ and $\pi_{Y}: \check{\mathcal{S}} \rightarrow Y$ are immersions, the map $\pi_{Y} \circ \pi_{X}{ }^{-1}: \pi_{X}(\Gamma) \rightarrow$ $\pi_{Y}(\Gamma)$ is an immersion as well. Thus it makes sense to use our graph notation, $\Gamma=\gamma \mid \delta$, for real curves, where $\gamma=\pi_{X}(\Gamma)$ and $\delta=\pi_{Y}(\Gamma)$. A real curve $\gamma \mid \delta$ is geometrically no different from the edge of a triangular graph; the projections $\gamma$ and $\delta$ are always straight lines. Any real curve is isometric with the graph of a real analytic function.

The three real curves which bound the triangular graph $P \mid Q$ generate a topological cell decomposition of $\bar{P} \mid \bar{Q}$ into vertices, edges, and the graph $P \mid Q$ itself. The generators of the edge group, $\sigma_{i j}$, acting on $\bar{P} \mid \bar{Q}$, generate three closed graphs, each having one edge in common with $\bar{P} \mid \bar{Q}$. By continuing this construction we obtain a cell decomposition of $[P \mid Q]$ into 2-cells isometric with $P \mid Q, 1$-cells isometric with one of the edges of $\bar{P} \mid \bar{Q}$, and points. The cell complex as a whole defines a tiling $\mathcal{T}$; the 2 -cells by themselves form a set of tiles, $\mathcal{T}_{2}$, and every element of $\mathcal{T}_{2}$ can be expressed as $g(P \mid Q)$, where $g$ is an element of the edge group, $G$.

To show that $G$ is the maximal isometry group we first need to check that the tiling $\mathcal{T}$ is primitive, that is, there is no refinement of the tiles $\mathcal{T}_{2}$ by additional real curves within $[P \mid Q]$ we may have missed. For this it suffices to check that there are no real curves within $P \mid Q$. Before we can prove this statement we need some basic properties of real curves.

Definition 2.10. A real curve is complete if it is not a proper subset of any other real curve.

Lemma 2.3. The closure in $\mathcal{S}$ of a complete real curve $\gamma \mid \delta \subset \mathcal{S}$, if bounded, has singular endpoints.

Proof. Without loss of generality let $\gamma$ and $\delta$ lie on the real axes of, respectively, $X$ and $Y$. The functions $f$ of the function elements $(U, f)$, which represent $\mathcal{S}$ locally, will then have power series on the real axis (of $X$ ) with real coefficients. Since a real power series when analytically continued along the real axis continues to be real, we can continue $\gamma \mid \delta$ until we encounter either a singularity of $f$ or a zero of $f^{\prime}$ (i.e. a singularity of $f^{-1}$ on the real axis of $Y$ ).

The next lemmas deal with the angles formed by intersecting real curves.

Definition 2.11. The angle between lines $\gamma$ and $\gamma^{\prime}$ (in $X$ or $Y$ ), denoted $\angle\left(\gamma, \gamma^{\prime}\right)$, is the smallest counterclockwise rotation required to make $\gamma$ parallel to $\gamma^{\prime}$. 
Lemma 2.4. If real curves $\gamma \mid \delta \subset \check{\mathcal{S}}$ and $\gamma^{\prime} \mid \delta^{\prime} \subset \check{\mathcal{S}}$ intersect, then $\angle\left(\gamma, \gamma^{\prime}\right)=\angle\left(\delta, \delta^{\prime}\right)$.

Proof. Near the point of intersection $\check{\mathcal{S}}$ is represented by a function element $(U, f)$ where $f$ is conformal. The equality of angles, formed by a pair of lines in $X$ and their images by $f$ in $Y$, is simply the geometrical statement that $f$ is conformal.

Lemma 2.5. Only a finite number $n>1$ of real curves can intersect at any point of a nontrivial Riemann surface and the angle formed by any pair must be a multiple of $\pi / n$.

Proof. Suppose $\gamma \mid \delta$ and $\gamma^{\prime} \mid \delta^{\prime}$ intersect with angle $\angle\left(\gamma, \gamma^{\prime}\right)=\angle\left(\delta, \delta^{\prime}\right)=\alpha>0$ on a nontrivial Riemann surface $\mathcal{S}$; for convenience, let $(0,0)$ be the point of intersection. These curves are fixed by Schwarz reflections $\sigma$ and $\sigma^{\prime}$, respectively, and $\sigma^{\prime} \sigma=r(2 \alpha, 2 \alpha)$ is an isometry of $\mathcal{S}$. The neighborhood of the point of intersection is the graph

$$
\mathcal{S}_{0}=\{(x, f(x)): x \in U\},
$$

where $U$ is a neighborhood of the origin in $X$, and $f$ is conformal at $x=0$. The Taylor series for $f$ at the origin has the form

$$
f(x)=\sum_{k=1}^{\infty} a_{k} x^{k}
$$

where $a_{1} \neq 0$. A short calculation shows

$$
r(2 \alpha, 2 \alpha) \mathcal{S}_{0}=\left\{\left(x, f_{\alpha}(x)\right): x \in U_{\alpha}\right\},
$$

where $U_{\alpha}=e^{i 2 \alpha} U$ is again a neighborhood of the origin, and

$$
f_{\alpha}(x)=\sum_{k=1}^{\infty} a_{k} e^{i 2 \alpha(1-k)} x^{k}
$$

Since $r(2 \alpha, 2 \alpha)$ is an isometry, the Taylor series for $f$ and $f_{\alpha}$ must agree, term by term. Now if $\alpha=\pi \omega$ and $\omega$ is irrational, then $\omega(1-k)$ can be an integer only for $k=1$ (so that $\left.e^{i 2 \alpha(1-k)}=1\right)$. However, this requires $a_{k}=0$ for $k>1$ which is impossible since $\mathcal{S}$ is nontrivial. Thus we may assume $\omega=p / q$ where $p$ and $q$ are relatively prime positive integers, $p<q$ (since $\alpha<\pi$ ), and $a_{1+m q} \neq 0$ for some integer $m>0$.

Now let $\gamma^{\prime \prime} \mid \delta^{\prime \prime}$ be any real curve that intersects $\gamma \mid \delta$ at the origin; then $\angle\left(\gamma, \gamma^{\prime \prime}\right)=$ $\angle\left(\delta, \delta^{\prime \prime}\right)=\pi\left(p^{\prime} / q^{\prime}\right)$ by the argument just given, where $p^{\prime}$ and $q^{\prime}$ are relatively prime positive integers, $p^{\prime}<q^{\prime}$. However, since $a_{1+m q} \neq 0$, we must have $e^{-i 2 \pi\left(p^{\prime} / q^{\prime}\right) m q}=1$, or that $q^{\prime}$ divides the product $m q=n$. This shows that $\angle\left(\gamma, \gamma^{\prime \prime}\right)$ is a multiple of $\pi / n$, for some $n>1$.

Definition 2.12. If exactly $n>1$ real curves intersect at a regular point $(x, y)$ of a Riemann surface, the integer $n$ is called the order of $(x, y)$.

Clearly, any triangular graph with a nontrivial isometry must be "isosceles" and fails 

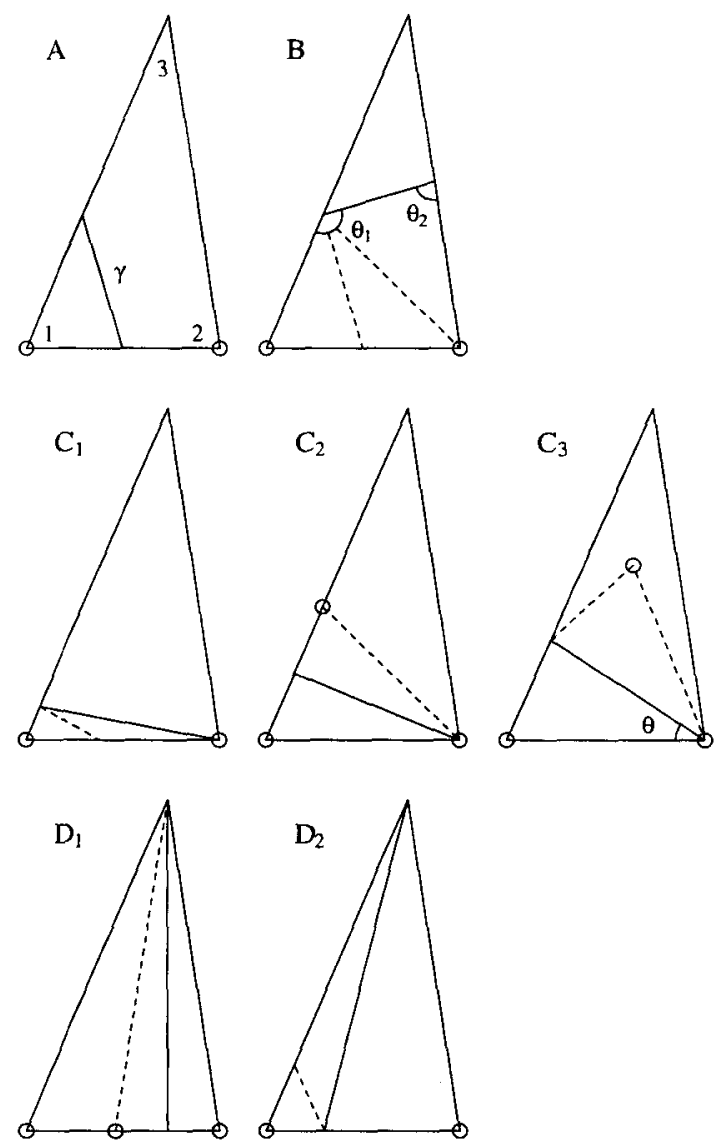

Fig. 2. Diagrams used in the proof of Lemma 2.6.

to be primitive because it can be decomposed into two isometric tiles. This is made precise by the following lemma.

Lemma 2.6. Let $P \mid Q$ be a nontrivial triangular graph with trivial isometry group, then $P \mid Q$ contains no real curves.

Proof. Suppose $P \mid Q$ contains a real curve and call its completion $\gamma \mid \delta$. We recall that $\gamma$, and its closure in $X, \bar{\gamma}$, are straight lines and $\bar{\gamma}$ cannot have an endpoint within $P$ (Lemma 2.3). The possible geometrical relationships between $\bar{\gamma}$ and $P$ are shown in Fig. 2. Since $P \mid Q$ is nontrivial, at least two vertices are singular and are shown circled in each diagram. Either $\bar{\gamma}$ intersects two edges of $P$, as in cases $\mathbf{A}$ and $\mathbf{B}$, or it intersects an edge and the opposite vertex which may be singular (case C) or possibly regular (case D). The vertex labels on the diagram refer to our notation for the vertex angles and edges. For example, $\alpha_{1}$ and $\beta_{1}$ are the angles in $P$ and $Q$, respectively, of vertex $1 ; P_{12} \mid Q_{12}$ is the edge (real curve) bounded by vertices 1 and 2 , etc. 
Case $\mathrm{A}$ is easily disposed of using Lemma 2.4:

$$
\begin{aligned}
\angle\left(\bar{\gamma}, P_{12}\right)=\angle\left(\bar{\gamma}, P_{13}\right)+\alpha_{1}=\angle\left(\bar{\delta}, Q_{13}\right)+\alpha_{1} & =\angle\left(\bar{\delta}, Q_{12}\right)-\beta_{1}+\alpha_{1} \\
& =\angle\left(\bar{\gamma}, P_{12}\right)-\beta_{1}+\alpha_{1} .
\end{aligned}
$$

This is impossible because vertex 1 is singular $\left(\alpha_{1} \neq \beta_{1}\right)$.

By using Schwarz reflection to imply the existence of additional real curves, the remaining cases either reduce to case $A$ or imply the existence of a singularity within $P \mid Q$ or one of its edges-neither of which is possible.

First consider case B. Let $\gamma$ intersect $P_{13}$ at $x_{1}$ and $P_{23}$ at $x_{2}$, forming angles $\theta_{1}$ and $\theta_{2}$ (see Fig. 2). Any other complete real curve with projection $\gamma^{\prime}$ which intersects $x_{1}$ makes a finite angle with $\gamma$ by Lemma 2.5. Thus we may assume $\theta_{1}$ is the smallest possible angle (for a $\gamma$ that intersects both $P_{13}$ and $P_{23}$ ), and similarly for $\theta_{2}$. Since one of $\theta_{1}$ and $\theta_{2}$ must be greater than $\pi / 2$, we assume without loss of generality it is $\theta_{1}$. If we now reflect $\gamma$ in $P_{13}$ we obtain a real curve with projection $\gamma^{\prime}$ such that $\gamma^{\prime}$ intersects $P_{13}$ but not $P_{23}$. Thus case B always reduces to cases $\mathrm{A}$ or $\mathrm{C}$.

In case $C$ we consider the sequence of real curves with projections $\gamma_{k}$, where $\gamma_{0}=P_{23}$, $\gamma_{1}=\gamma$, and $\gamma_{k+1}$ is the image of $\gamma_{k-1}$ under reflection in $\gamma_{k}$. Let $\theta_{k}$ be the angle formed at vertex 2 in $P$ by $\gamma_{k}$. Clearly, for some $k$ we arrive at a $\gamma^{\prime}=\gamma_{k}$ such that $\theta=\theta_{k} \leq \alpha_{2} / 2$ (see Fig. 2). This leads to three subcases: $\mathrm{C}_{1}$, where $\angle\left(\gamma^{\prime}, P_{13}\right) \leq \pi / 2, \mathrm{C}_{2}$, where $\gamma^{\prime}$ and $P_{13}$ are perpendicular, and $\mathrm{C}_{3}$, where $\angle\left(\gamma^{\prime}, P_{13}\right) \geq \pi / 2$. In case $\mathrm{C}_{2}, \theta<\alpha_{2} / 2$ since otherwise $P \mid Q$ would have a nontrivial isometry (reflection in $\gamma^{\prime} \mid \delta^{\prime}$ ). All three subcases immediately lead to contradictions. In $\mathrm{C}_{1}$, reflecting $\gamma^{\prime}$ in $P_{13}$ presents us with a $\gamma^{\prime \prime}$ satisfying case $\mathrm{A}$. In $\mathrm{C}_{3}$, the image of vertex 1 under reflection in $\gamma^{\prime}$ implies a singularity within $P$; in $\mathrm{C}_{2}$ the same reflection implies a singularity on $P_{13}$.

Case $\mathrm{D}$ : we either have $\angle\left(\gamma, P_{12}\right)=\pi / 2$, case $\mathrm{D}_{1}$, or $\angle\left(\gamma, P_{12}\right) \neq \pi / 2$, case $\mathrm{D}_{2}$. Since $P$ has no nontrivial isometry, a reflection in $\gamma$ in case $D_{1}$ would place the image of either vertex 1 or 2 (both singular) somewhere on $P_{12}$. In $D_{2}$, a Schwarz reflection of $\gamma$ leads to case $\mathrm{A}$.

Theorem 2.7. Let $P \mid Q$ be a nontrivial triangular graph with trivial isometry group, then the maximal isometry group of $[P \mid Q]$ is the edge group of $P \mid Q$.

Proof. We use the real curves to decompose $[P \mid Q]$ into a set of tiles (2-cells) $\mathcal{T}_{2}$. Lemma 2.6 tells us that $P \mid Q \in \mathcal{T}_{2}$, so that $\mathcal{T}_{2}=G(P \mid Q)$, where $G$ is the edge group of $P \mid Q$. On the other hand, if $h$ is an isometry of $[P \mid Q]$, then $h(P \mid Q)=P^{\prime} \mid Q^{\prime} \in \mathcal{T}_{2}$, where $P^{\prime} \mid Q^{\prime}=g(P \mid Q)$ for some $g \in G$. However, since $P \mid Q$ has no nontrivial isometries, the map $h^{-1} g: P|Q \rightarrow P| Q$ must be the identity and $h=g$.

We conclude this section with a formula for the topological genus of a Riemann surface $[P \mid Q]$ compactified by the translation subgroup of its isometry group, the lattice group $\Lambda$ of $[P \mid Q]$.

Definition 2.13. The vertex groups $G_{i}(i=1,2,3)$ of a triangular graph $P \mid Q$, are the subgroups of the edge group of $P \mid Q$ generated by the adjacent edges of, respectively, the three vertices of $P \mid Q$. 
Theorem 2.8. Let $P \mid Q$ be a nontrivial triangular graph with trivial isometry group. Let $G$ be the isometry group of $[P \mid Q]$, let $\Lambda$ be its lattice group, and let $G_{i}(i=1,2,3)$ be the three vertex groups of $P \mid Q$. If $|G / \Lambda|$ is finite, the genus $g$ of the surface $[P \mid Q] / \Lambda$ satisfies

$$
2-2 g=|G / \Lambda|\left(\sum_{i=1}^{3} \frac{1}{\left|G_{i}\right|}-\frac{1}{2}\right)
$$

Proof. If $|G / \Lambda|$ is finite we can view $[P \mid Q] / \Lambda$ as a finite cell complex. We can relate the number of 0-cells, $N_{0}$, and the number of 1-cells, $N_{1}$, in this complex to the number of 2-cells, $N_{2}$. Since every 2 -cell is bounded by three 1-cells, each of which bounds exactly one other 2-cell, $N_{1}=\frac{3}{2} N_{2}$. Similarly, the boundary of each 2-cell contains three 0 -cells (the vertices $i=1,2,3$ ), each of which belongs to the boundary of a number of 2-cells equal to the order of the corresponding vertex group, $\left|G_{i}\right|$. Thus $N_{0}=\left(\sum_{i=1}^{3}\left|G_{i}\right|^{-1}\right) N_{2}$. Finally, since $P \mid Q$ has no nontrivial isometry, and $G / \Lambda$ acts transitively on the 2-cells of $[P \mid Q] / \Lambda, N_{2}=|G / \Lambda|$. The result (22) follows from Euler's formula, $2-2 g=N_{2}-N_{1}+N_{0}$.

\subsection{Discreteness and Uniformity}

The whole point of immersing a Riemann surface $\mathcal{S}$ in $X \times Y=\mathbb{C}^{2}$ is that by forming sections of $\mathcal{S}$, i.e. intersections with $X=\mathbb{C}$, one obtains patterns of points. A very primitive property of a point set, normally taken for granted in crystallography, is discreteness.

Definition 2.14. The section of the Riemann surface $\mathcal{S}$ at $x$ is the set-valued map

$$
\mathcal{A}(x)=\pi_{Y} \circ \pi_{X}^{-1}(x) \text {. }
$$

The basic property of a holomorphic function, that its zeros form a discrete set, translates to the statement that the preimages $\pi_{X}{ }^{-1}(x)$ are discrete in $\mathcal{S}$. We use the stronger property that $\pi_{X}{ }^{-1}(x)$ is discrete in $X \times Y$ to define a discrete Riemann surface. This is equivalent to the following statement about sections:

Definition 2.15. A Riemann surface $\mathcal{S}$ is discrete iff its sections $\mathcal{A}(x)$ are discrete in $Y$ for every $x \in X$.

All the statements we can make about discreteness of a Riemann surface $\mathcal{S}$ hinge upon properties of the lattice group of $\mathcal{S}, \Lambda$. One property is the rank, $\operatorname{rk}(\Lambda)$, given by the cardinality of the generators of $\Lambda$. The orbit of the origin of $X \times Y, \Lambda(0,0)$, is called a lattice and is also represented by the symbol $\Lambda$. When $\operatorname{rk}(\Lambda)=4$, a second property is the determinant of the lattice, $\operatorname{det} \Lambda$. If $\operatorname{det} \Lambda>0$, the four generators of $\Lambda$ are linearly independent (as vectors in $X \times Y$ ); if det $\Lambda=0$ the generators are linearly dependent and $\Lambda$ (as a lattice) is not discrete in $X \times Y$. A lattice with $\operatorname{rk}(\Lambda)>4$ is never discrete in $X \times Y$.

Notation. The standard measure for a set $A$ is written $|A|$. If $A$ is a region in $\mathbb{C}$, then $|A|$ is its area; if $A$ is a set of points, then $|A|$ is its cardinality. Finally, if $\Lambda$ is a rank 4 lattice, then $|\Lambda|=\sqrt{\operatorname{det} \Lambda}$ is the volume in $X \times Y$ of its fundamental region. 
The following lemma provides a necessary condition for discreteness:

Lemma 2.9. The lattice group $\Lambda$, of a discrete, nontrivial Riemann surface $\mathcal{S}$, is discrete (as a lattice) in $X \times Y$ and in particular, $\mathrm{rk} \Lambda \leq 4$.

Proof. If $\Lambda$ is not discrete we can find a sequence of $t(u, v) \in \Lambda$ such that both $\|u\| \rightarrow 0$ and $\|v\| \rightarrow 0$. Let $\left(x_{0}, y_{0}\right)$ be a regular point of $\mathcal{S}$, then $y_{0} \in \mathcal{A}\left(x_{0}\right)$. Near $\left(x_{0}, y_{0}\right)$ we can represent $\mathcal{S}$ by the graph

$$
U \mid V=\{(x, f(x)): x \in U\}
$$

where $U$ is a neighborhood of $x_{0}$ in $X, f$ is conformal in $U$, and $f\left(x_{0}\right)=y_{0}$. Since $t(u, v)$ is an isometry, $t(u, v)(U \mid V) \subset \mathcal{S}$. From

$$
t(u, v)(U \mid V)=\{(x+u, f(x)+v): x \in U\},
$$

we see that $f\left(x_{0}-u\right)+v \in \mathcal{A}\left(x_{0}\right)$, since $x_{0}-u \in U$ as $\|u\|$ can be arbitrarily small. Since $\mathcal{S}$ is discrete, $y_{0}$ is isolated in $Y$ and there must be a subsequence $t\left(u^{\prime}, v^{\prime}\right)$ such that $f\left(x_{0}-u^{\prime}\right)+v^{\prime}=f\left(x_{0}\right)$. If, within the sequence $t\left(u^{\prime}, v^{\prime}\right)$, there is a subsequence $t\left(u^{\prime \prime}, v^{\prime \prime}\right)$ with $u^{\prime \prime}=0$, then $v^{\prime \prime}=f\left(x_{0}\right)-f\left(x_{0}-u^{\prime \prime}\right)=0$ and we have a contradiction. Thus there must be a subsequence with $u^{\prime \prime} \neq 0$. Since $f$ is conformal at $x_{0}$,

$$
\begin{aligned}
\lim _{u^{\prime \prime} \rightarrow 0} \frac{f\left(x_{0}\right)-f\left(x_{0}-u^{\prime \prime}\right)}{u^{\prime \prime}} & =f^{\prime}\left(x_{0}\right) \\
& =\lim _{\left(u^{\prime \prime}, v^{\prime \prime}\right) \rightarrow(0,0)} \frac{v^{\prime \prime}}{u^{\prime \prime}} .
\end{aligned}
$$

However, the second limit, above, is independent of $x_{0}$ so we are forced to conclude that $f^{\prime}$ is constant. This is impossible because $\mathcal{S}$ is nontrivial.

The point sets studied in crystallography normally are Delone sets and have the property of being uniformly discrete [4]. For a point set in $\mathbb{R}^{n}$ this means there exists a real number $r>0$ such that a spherical neighborhood of radius $r$ about any point of the set contains no other point of the set. For the sets $\mathcal{A}(x)$ generated by Riemann surfaces this property is clearly too strong: it is violated whenever $x$ is near a branch point of $\pi_{X}$. We therefore adopt a weaker form of this property which is nevertheless stronger than discreteness and useful in establishing the existence of the density.

Definition 2.16. Let $B_{r}(y) \subset Y$ be an open disk of radius $r$ centered at $y$. A Riemann surface $\mathcal{S}$, with sections $\mathcal{A}(x)$, is finitely discrete if for some $r>0,\left|\mathcal{A}(x) \cap B_{r}(y)\right|$ is uniformly bounded above for all $x \in X$ and $y \in Y$.

Since a disk of radius $r^{\prime}$ can always be covered by finitely many disks of radius $r$, the finitely discrete property holds for any $r^{\prime}$ once it has been established for a particular $r$.

We now introduce the class of Riemann surfaces which is the focus of this study.

Definition 2.17. A Riemann surface is crystallographic if its lattice group $\Lambda$ has rank 4 and $|\Lambda|>0$. 
Definition 2.18. Any isometry $g$ of $X \times Y$ can be uniquely expressed in the form $g=g_{0} \lambda$, where $g_{0}$ fixes the origin and $\lambda$ is a translation. The derived point group of the isometry group $G, \psi(G)$, is the image of $G$ by the homomorphism $\psi: g \mapsto g_{0}$. Since $\operatorname{Ker} \psi=\Lambda$, the lattice group of $G$, we have the isomorphism $\psi(G) \simeq G / \Lambda$.

It is important to remember that $\psi(G)$ need not be a subgroup of $G$; nevertheless, the lattice group of $G$ is always left invariant by $\psi(G)$, just as it is invariant within $G$. Furthermore, if a Riemann surface is crystallographic, then the action (by conjugation) of $\psi(G)$ on its lattice group $\Lambda$ is a faithful representation of $G / \Lambda$. Since the isometry group of a (finite rank) lattice is finite, we have that $G / \Lambda$, for a crystallographic Riemann surface, is always finite.

Lemma 2.10. A Riemann surface determined by a triangular graph $P \mid Q$, if crystallographic, is finitely discrete.

Proof. Let $B_{r}(x, y) \subset X \times Y$ be an open ball of radius $r$ centered at an arbitrary point $(x, y)$. Consider the piece of the Riemann surface within this ball, $\mathcal{S}_{B}=[P \mid Q] \cap$ $B_{r}(x, y)$, and the projection $\pi_{X}: \mathcal{S}_{B} \rightarrow X .[P \mid Q]$ is finitely discrete if there is a uniform upper bound on the number of preimages $\pi_{X}^{-1}(x)$.

$[P \mid Q]$ is covered by the orbit of closed graphs, $G(\bar{P} \mid \bar{Q})$, where $G$ is the edge group of $P \mid Q$. Let $\Lambda$ be the lattice group of $G$, then $G(\bar{P} \mid \bar{Q})$ is the union of cosets, $H_{i}(\bar{P} \mid \bar{Q})$, $i=1, \ldots, N$, where $N=|G / \Lambda|$ is finite because $[P \mid Q]$ is crystallographic. Again, because $[P \mid Q]$ is crystallographic, all but finitely many graphs in $H_{i}(\bar{P} \mid \bar{Q})$ have empty intersection with a ball of radius $r$, in particular, $B_{r}(x, y)$. Thus we have a bound (independent of $x$ and $y)$ on the number of graphs in $G(\bar{P} \mid \bar{Q})$ which intersect $B_{r}(x, y)$. However, a graph can have at most one preimage of $\pi_{X}$; hence $\left|\pi_{X}^{-1}(x)\right|$ is uniformly bounded above.

The sections $\mathcal{A}(x)$ of a crystallographic Riemann surface also possess a uniformity with respect to the parameter $x$. Our handle on this property is provided, in part, by the smooth behavior of $\mathcal{A}(x)$ with $x$. Before we can proceed, however, we need to be aware of two point sets in $X$ which create problems: branch points (of $\pi_{X}$ ) and crossing points.

Definition 2.19. A point $(x, y) \in \mathcal{S}$ is a self-intersection point if in the description of $\mathcal{S}$ as a complete global analytic function there exist function elements $(U, f)$ and $(V, g)$, such that $x \in U \cap V, f \neq g$ in $U \cap V$, and $f(x)=g(x)=y$. The point $x \in X$ is called a crossing point.

Lemma 2.11. A Riemann surface determined by a triangular graph $P \mid Q$ has countably many self-intersection points and $\pi_{X}$ has countably many branch points.

Proof. $[P \mid Q]$ is the union of countably many closed graphs $\bar{P}_{i} \mid \bar{Q}_{i}$ given by the orbit of $\bar{P} \mid \bar{Q}$ under the action of the edge group. Since each graph has at most three singular points, $\pi_{X}$ has countably many branch points. If there were uncountably many selfintersection points, then uncountably many must arise from one pair of distinct graphs, 
say $\bar{P}_{i} \mid \bar{Q}_{i}$ and $\bar{P}_{j} \mid \bar{Q}_{j}$. Let $f_{i}$ and $f_{j}$ be the corresponding conformal maps; then $f_{i}(x)=$ $f_{j}(x)$ would have uncountably many solutions $x \in \bar{P}_{i} \cap \bar{P}_{j}$. Thus either $f_{i}=f_{j}$, a contradiction, or the zeros of $f_{i}-f_{j}$ would not be isolated, another impossibility.

Lemma 2.12. Let $[P \mid Q]$ be crystallographic, let $X_{c} \subset X$ be its crossing points, and let $X_{b} \subset X$ be the branch points of $\pi_{X}$; then for any pair $x, x^{\prime} \in X \backslash\left(X_{b} \cup X_{c}\right)$, there exists a bijection of sections of $[P \mid Q]$,

$$
\Psi: \mathcal{A}(x) \rightarrow \mathcal{A}\left(x^{\prime}\right)
$$

such that $\|y-\Psi(y)\|$ is uniformly bounded above for $y \in \mathcal{A}(x)$.

Proof. We arrive at $\Psi$ by composing bijections

$$
\begin{aligned}
& \Psi_{1}: \mathcal{A}(x) \rightarrow \mathcal{A}\left(x^{\prime \prime}\right), \\
& \Psi_{2}: \mathcal{A}\left(x^{\prime \prime}\right) \rightarrow \mathcal{A}\left(x^{\prime}\right),
\end{aligned}
$$

such that $\left\|y-\Psi_{1}(y)\right\|$ and $\left\|y^{\prime \prime}-\Psi_{2}\left(y^{\prime \prime}\right)\right\|$ are (correspondingly) uniformly bounded. The lemma then follows by application of the triangle inequality.

Since $[P \mid Q]$ is crystallographic, we can partition $X \times Y$ into translates of a bounded fundamental region, $V(0)$, of its lattice $\Lambda$. Thus for any pair $x, x^{\prime} \in X$ we can write

$$
\begin{aligned}
(x, 0) & \in V(0)+\lambda, \\
\left(x^{\prime}, 0\right) & \in V(0)+\lambda^{\prime},
\end{aligned}
$$

where $\lambda, \lambda^{\prime} \in \Lambda$. Consider the point

$$
\left(x^{\prime \prime}, y^{\prime \prime}\right)=(x, 0)+\lambda^{\prime}-\lambda \in V(0)+\lambda^{\prime} .
$$

Since

$$
\left(x^{\prime \prime}-x^{\prime}, y^{\prime \prime}\right)=\left(x^{\prime \prime}, y^{\prime \prime}\right)-\left(x^{\prime}, 0\right) \in V(0)-V(0),
$$

both $\left\|x^{\prime \prime}-x^{\prime}\right\|$ and $\left\|y^{\prime \prime}\right\|$ have upper bounds independent of $x$ and $x^{\prime}$. Now

$$
\mathcal{A}(x)=\{y \in Y:(x, y) \in[P \mid Q]\}
$$

and

$$
\mathcal{A}(x)+y^{\prime \prime}=\left\{y^{\prime} \in Y:\left(x, y^{\prime}-y^{\prime \prime}\right) \in[P \mid Q]\right\} .
$$

However, $\left(x, y^{\prime}-y^{\prime \prime}\right) \in[P \mid Q]$ iff $\left(x, y^{\prime}-y^{\prime \prime}\right)+\lambda^{\prime \prime} \in[P \mid Q]$, where $\lambda^{\prime \prime} \in \Lambda$. Choosing

$$
\lambda^{\prime \prime}=\lambda^{\prime}-\lambda=\left(x^{\prime \prime}-x, y^{\prime \prime}\right)
$$

we obtain

$$
\mathcal{A}(x)+y^{\prime \prime}=\left\{y^{\prime} \in Y:\left(x^{\prime \prime}, y^{\prime}\right) \in[P \mid Q]\right\}=\mathcal{A}\left(x^{\prime \prime}\right) .
$$

As our first bijection we take the translation $\Psi_{1}(y)=y+y^{\prime \prime}$, where $\left\|y^{\prime \prime}\right\|$ is uniformly bounded from above. The point of this intermediate step is that for $\Psi_{2}$ we need consider only pairs of sections with bounded separation $\left\|x^{\prime \prime}-x^{\prime}\right\|$. 
In constructing $\Psi_{2}$ we avoid branch points and crossing points. Since $x \in X \backslash\left(X_{b} \cup\right.$ $\left.X_{c}\right)$, (33) implies $x^{\prime \prime} \in X \backslash\left(X_{b} \cup X_{c}\right)$. Let $\gamma:[0,1] \rightarrow X \backslash\left(X_{b} \cup X_{c}\right)$ be a smooth rectifiable curve with $\gamma(0)=x^{\prime \prime}$ and $\gamma(1)=x^{\prime}$. To show that $\gamma$ exists we recall that $X_{b} \cup X_{c}$ is countable. We can then find $\gamma$ in the uncountable family of circular arcs with endpoints $x^{\prime \prime}$ and $x^{\prime}$, since each point of $X_{b} \cup X_{c}$ can eliminate at most one arc.

The curve $\gamma(t)$ generates a homotopy of the sections $\mathcal{A}\left(x^{\prime \prime}\right)$ and $\mathcal{A}\left(x^{\prime}\right)$. At each point $y^{\prime \prime} \in \mathcal{A}\left(x^{\prime \prime}\right), \gamma(t)$ is lifted to a unique curve $\gamma(t) \mid \delta(t) \subset[P \mid Q]$ with endpoint $(\gamma(0), \delta(0))=\left(x^{\prime \prime}, y^{\prime \prime}\right)$ and we define our second bijection by $\Psi_{2}\left(y^{\prime \prime}\right)=\delta(1)$. To finish the proof we need to show that $\|\delta(1)-\delta(0)\|$ is uniformly bounded.

Let $\check{P}$ be a closed subset of $\bar{P}$ from which suitably small neighborhoods of all the branch points of $\pi_{X}$ have been removed. Let $\check{P}|\check{Q} \subset \bar{P}| \bar{Q}$ be the corresponding graph. The orbit under the edge group, $\check{\mathcal{S}}=G(\check{P} \mid \check{Q}) \subset[P \mid Q]$, is a Riemann surface from which all the points of ramification (of the map $\pi_{X}$ ) have been "cut out". The complement, $\hat{\mathcal{S}}=[P \mid Q] \backslash \mathcal{S}$, is the disjoint union of the branched neighborhoods of all the points of ramification. It is possible to find curves $\gamma(t)$, such as the circular arcs considered above, where the branched neighborhoods $\hat{\mathcal{S}}_{i}$ visited by $\gamma(t) \mid \delta(t)$ are visited only once, for $t \in T_{i} \subset[0,1]$. Also, because we can bound the length $L$ of $\gamma$ and there is a minimum distance between branch points (on the branched covering of $X$ ), the number of such subintervals $T_{i}$ is bounded, i.e. $i=1, \ldots, N$. The bounds on $N$ and $L$ are uniform bounds, independent of the points $x$ and $x^{\prime}$.

Two additional bounds are needed before we can proceed to bound $\|\delta(1)-\delta(0)\|$. The first is an upper bound $D$ on the diameter of the projection of a branched neighborhood, $\pi_{Y}\left(\hat{\mathcal{S}}_{i}\right)$. This follows from the fact that $\hat{\mathcal{S}}_{i}$ is isometric with the branched neighborhood $\hat{\mathcal{S}}_{j}$ of a vertex of $\bar{P} \mid \bar{Q}$, and $\hat{\mathcal{S}}_{j} \subset G_{j}(P \mid Q)$, where $G_{j}$ is the corresponding vertex group. Clearly the maximum diameter of $\pi_{Y}\left(G_{j}(P \mid Q)\right)$ is bounded because $Q$ is bounded.

The map $f: P \rightarrow Q$ (which defines $P \mid Q)$, when restricted to $\check{P}$ is conformal and $\left\|f^{\prime}\right\|$ has a maximum value, $\mu$, since $\check{P}$ is closed. This means that if $\gamma(t)|\delta(t) \in \check{P}| \check{Q}$, then

$$
\left\|\frac{d \delta}{d t}\right\|=\left\|f^{\prime}(\gamma) \frac{d \gamma}{d t}\right\| \leq \mu\left\|\frac{d \gamma}{d t}\right\| .
$$

Because $\check{\mathcal{S}}$ is generated from $\check{P} \mid \check{Q}$ by the action of $G$, this bound applies globally, for $\gamma(t) \mid \delta(t) \in \check{\mathcal{S}}$.

We are now ready to complete the proof:

$$
\|\delta(1)-\delta(0)\|=\left\|\int_{[0,1]} \frac{d \delta}{d t}\right\| \leq\left\|\int_{\cup_{i=1}^{N} T_{i}} \frac{d \delta}{d t}\right\|+\left\|\int_{[0,1] \backslash \cup_{i=1}^{N} T_{i}} \frac{d \delta}{d t}\right\| .
$$

For each piece of the curve in a branched neighborhood we have

$$
\left\|\int_{T_{i}} \frac{d \delta}{d t}\right\| \leq D
$$

while in the complement $(\check{\mathcal{S}})$,

$$
\left\|\int_{[0,1] \backslash \cup_{i=1}^{N} T_{i}} \frac{d \delta}{d t}\right\| \leq \int_{[0,1] \backslash \cup_{i=1}^{N} T_{i}}\left\|\frac{d \delta}{d t}\right\|
$$




$$
\begin{aligned}
& \leq \mu \int_{[0,1] \backslash \cup_{i=1}^{N} T_{i}}\left\|\frac{d \gamma}{d t}\right\| \\
& \leq \mu L .
\end{aligned}
$$

Inequality (40) thus becomes

$$
\|\delta(1)-\delta(0)\| \leq N D+\mu L .
$$

For discrete Riemann surfaces with sufficiently uniform sections $\mathcal{A}(x)$, one can define their density.

Definition 2.20. Let $B_{R}(0) \subset Y$ be a disk of radius $R$ centered at the origin. The limit

$$
\rho(x)=\lim _{R \rightarrow \infty} \frac{\left|B_{R}(0) \cap \mathcal{A}(x)\right|}{\left|B_{R}(0)\right|},
$$

if it exists and is finite, is the density of $\mathcal{A}(x)$.

With the aid of Lemmas 2.10 and 2.12 we can show that, for a crystallographic Riemann surface generated by a triangular graph, $\rho(x)$ exists and is (essentially) independent of $x$.

Notation. The standard volume form in $X$ is $\omega_{X}=d x \wedge d \bar{x}$, its pullback on a Riemann surface $\mathcal{S}$ is written $\pi_{X}{ }^{*} \omega_{X}$.

Theorem 2.13. If $[P \mid Q]$ is crystallographic with lattice group $\Lambda$, its sections $\mathcal{A}(x)$ have density

$$
\rho=\frac{1}{|\Lambda|} \int_{[P \mid Q] / \Lambda} \pi_{X}{ }^{*} \omega_{X},
$$

independent of $x$, provided $x$ is not a crossing point or a branch point of $\pi_{X}$. If $G$ is the edge group of $P \mid Q$, then

$$
\rho=\frac{|G / \Lambda||P|}{|\Lambda|}
$$

Proof.

Notation. The expression $c=O(1 / R)$ indicates there exist constants $c_{1}$ and $c_{2}$ (independent of $R$ ) such that for sufficiently large $R, c_{1} / R<c<c_{2} / R$.

Let $B_{R}(0) \subset Y$ be a disk of radius $R$ centered at the origin and let

$$
N_{R}(x)=\left|B_{R}(0) \cap \mathcal{A}(x)\right|
$$

We first obtain a bound on the difference, $N_{R}(x)-N_{R}\left(x^{\prime}\right)$, when neither $x$ nor $x^{\prime}$ is a crossing point or a branch point of $\pi_{X}$. By Lemma 2.12 there exists a bijection 
$\Psi: \mathcal{A}(x) \rightarrow \mathcal{A}\left(x^{\prime}\right)$ such that if $y \in B_{R}(0) \cap \mathcal{A}(x)$, then $\Psi(y) \in B_{R+d}(0) \cap \mathcal{A}\left(x^{\prime}\right)$, where $d>0$ is a constant independent of $R, x$, and $x^{\prime}$. This shows

$$
\begin{aligned}
N_{R}(x) & \leq\left|B_{R+d}(0) \cap \mathcal{A}\left(x^{\prime}\right)\right| \\
& =N_{R}\left(x^{\prime}\right)+\left|\left(B_{R+d}(0) \backslash B_{R}(0)\right) \cap \mathcal{A}\left(x^{\prime}\right)\right| .
\end{aligned}
$$

We can cover the annulus $B_{R+d}(0) \backslash B_{R}(0)$ by $M_{R}$ disks $B_{r}\left(y^{\prime}\right)$ of a fixed radius $r>0$, where, for sufficiently large $R, M_{R}<m R$ and $m$ is a constant independent of $R$. By Lemma 2.10, $\left|B_{r}\left(y^{\prime}\right) \cap \mathcal{A}\left(x^{\prime}\right)\right|<n$, where $n$ is independent of $x^{\prime}$ and $y^{\prime}$. Thus $N_{R}(x)-$ $N_{R}\left(x^{\prime}\right)<m n R$. Combining this bound with the bound obtained by interchanging $x$ and $x^{\prime}$, we arrive at the statement

$$
N_{R}(x)-N_{R}\left(x^{\prime}\right)=\left|B_{R}(0)\right| O(1 / R) .
$$

We now introduce a disk $C_{R}(0) \subset X$ and consider the region $W_{R}=C_{R}(0) \times B_{R}(0) \subset$ $X \times Y$. Since the set of branch points and crossing points is countable and has zero measure in $X$, and $\pi_{X}$ is otherwise smooth,

$$
\int_{\pi_{X}\left([P \mid Q] \cap W_{R}\right)} \omega_{X}=\int_{[P \mid Q] \cap W_{R}} \pi_{X}^{*} \omega_{X}
$$

Because $[P \mid Q]$ is crystallographic, we can partition $X \times Y$ into translates of a bounded fundamental region of its lattice, $V(0)$. Let $\pi_{\Lambda}:[P \mid Q] \rightarrow[P \mid Q] / \Lambda$ be the standard projection on the quotient. On $[P \mid Q] \cap V(0)$ the map $\pi_{\Lambda}$ is 1 -to-1 and

$$
\int_{[P \mid Q] \cap V(0)} \pi_{X}^{*} \omega_{X}=\int_{[P \mid Q] / \Lambda}\left(\pi_{\Lambda}^{-1}\right)^{*} \pi_{X}^{*} \omega_{X}=\rho|\Lambda|
$$

This defines $\rho$, which we can make positive by an appropriate choice of orientation on $[P \mid Q]$.

Turning now to the region $W_{R}$, there is a maximal subset $\Lambda_{-} \subset \Lambda$ such that $\Lambda_{-}+$ $V(0) \subset W_{R}$ and a smallest subset $\Lambda_{+} \subset \Lambda$ such that $W_{R} \subset \Lambda_{+}+V(0)$. If $\lambda \in \Lambda_{+} \backslash \Lambda_{-}$, then $V_{\lambda}=(\lambda+V(0)) \cap W_{R}$ is a proper subset of a fundamental region and

$$
0<\int_{[P \mid Q] \cap V_{\lambda}} \pi_{X}^{*} \omega_{X}<\rho|\Lambda| .
$$

From this it follows that

$$
\rho\left|\Lambda_{-}\right||\Lambda|<\int_{[P \mid Q] \cap W_{R}} \pi_{X}{ }^{*} \omega_{X}<\rho\left|\Lambda_{+}\right||\Lambda|,
$$

and, from straightforward estimates of $\Lambda_{+}$and $\Lambda_{-}$, we conclude

$$
\int_{[P \mid Q] \cap W_{R}} \pi_{X}{ }^{*} \omega_{X}=\rho\left|W_{R}\right|(1+O(1 / R)) .
$$

The projection $\pi_{X}\left([P \mid Q] \cap W_{R}\right)$ covers the disk $C_{R}(0)$ multiple times, the multiplicity at the point $x \in C_{R}(0)$ being the number $N_{R}(x)$ defined above. Thus

$$
\int_{\pi_{X}\left([P \mid Q] \cap W_{R}\right)} \omega_{X}=\int_{C_{R}(0)} N_{R}(x) \omega_{X} .
$$


We can again neglect the countable set of branch points $X_{b}$ and crossing points $X_{c}$ to argue, for $x_{0} \in X \backslash\left(X_{b} \cup X_{c}\right)$ fixed,

$$
\begin{aligned}
\int_{C_{R}(0)} N_{R}(x) \omega_{X} & =\int_{C_{R}(0)} N_{R}\left(x_{0}\right) \omega_{X}+\int_{C_{R}(0)}\left(N_{R}(x)-N_{R}\left(x_{0}\right)\right) \omega_{X} \\
& =N_{R}\left(x_{0}\right)\left|C_{R}(0)\right|+\left|C_{R}(0)\right|\left|B_{R}(0)\right| O(1 / R),
\end{aligned}
$$

where in the last step we used (52). Combining (53), (57)-(59), and using $\left|W_{R}\right|=$ $\left|B_{R}(0)\right|\left|C_{R}(0)\right|$, we obtain

$$
\frac{N_{R}\left(x_{0}\right)}{\left|B_{R}(0)\right|}=\rho(1+O(1 / R)),
$$

and thus

$$
\lim _{R \rightarrow \infty} \frac{N_{R}\left(x_{0}\right)}{\left|B_{R}(0)\right|}=\rho
$$

To evaluate $\rho$ from (54), we regard $[P \mid Q] / \Lambda$ as $|G / \Lambda|$ equivalence classes of tiles, all isometric to $P \mid Q$. The result (48) follows because the integral of the form $\pi_{X}{ }^{*} \omega_{X}$ over $P \mid Q$ is just the volume of $\pi_{X}(P \mid Q)=P$ in $X$.

Formula (47) for the density was introduced by Kalugin [5] to extend the notion of stoichiometry to quasicrystals. Because the 2-form $\pi_{X}{ }^{*} \omega_{X}$ is closed, this formula gives the same density for 2 -manifolds homologous in the torus $(X \times Y) / \Lambda$. One must remember, however, that this homology invariant only corresponds to the true density when the map $\pi_{X}$ is orientation preserving (see (58)).

\section{Classification of Discrete Riemann Surfaces Generated by Conformal Maps of Right Triangles}

\subsection{Conformal Maps of Right Triangles}

The simplest nontrivial conformal maps of triangles, $f: P \rightarrow Q$, are those where one of the vertices of the corresponding graph $P \mid Q$ is regular. The edges of $P \mid Q$ adjacent to this vertex are real curves, and, by Lemma 2.5, belong to a set of $n>1$ real curves intersecting at the same vertex with minimum angle $\pi / n$. This suggests that among these, the simplest cases are the conformal maps with one regular vertex of order $2(n=2)$, i.e. conformal maps of right triangles with the right angle being the regular vertex.

Without loss of generality, we give $P$ and $Q$ a standard scale, position, and angular orientation as specified by the vertices $x_{i} \mid y_{i}$ of the corresponding graph $P \mid Q$ :

$$
\begin{aligned}
& x_{1}\left|y_{1}=0\right| 0, \\
& x_{2}\left|y_{2}=\cos \alpha\right| \cos \beta, \\
& x_{3}\left|y_{3}=e^{i \alpha}\right| e^{i \beta},
\end{aligned}
$$

where $0<\alpha<\pi / 2$ and $0<\beta<\pi / 2$ are two free real parameters. Below we frequently use the abbreviations $a=\cos \alpha, b=\cos \beta . P$ and $Q$ have angles $\alpha$ and $\beta$, respectively, 
at vertex 1 , and the corresponding complementary angles at vertex 3 . A nontrivial graph $P \mid Q$ has $\alpha \neq \beta$ with vertices 1 and 3 singular; vertex 2 is regular.

The Schwarz-Christoffel formula [10] gives the conformal map $f$ as the composition $f=h \circ g^{-1}$ where $g$ and $h$ map the upper half-plane of $Z=\mathbb{C}$ conformally onto, respectively, $P$ and $Q$. Explicitly:

$$
\begin{aligned}
& g(z)=A \int_{0}^{z} z^{\alpha / \pi-1}(1-z)^{-1 / 2} d z, \\
& h(z)=B \int_{0}^{z} z^{\beta / \pi-1}(1-z)^{-1 / 2} d z .
\end{aligned}
$$

In both (63) and (64) the branches of the fractional powers are chosen so that the integrands are real and positive for $z \in(0,1)$. The normalization factors $A$ and $B$ are positive real numbers determined by the conditions $g(1)=a$ and $h(1)=b$. Further properties of $g$ and $h$ are easily checked, in particular, $g(\infty)=e^{i \alpha}$ and $h(\infty)=e^{i \beta}$.

\subsection{Isometry Groups}

Notation. Let $K$ be a set of elements of a group $G$, and let $k \in G$ be some element. We denote by $\langle K\rangle$ the subgroup generated by the elements of $K$, and by $\{k\}_{G}$ the conjugacy class of $k$ in $G$.

In the case of right triangles, $P \mid Q$ can have a nontrivial isometry only if $\alpha=\pi / 4$ and $\beta=\pi / 4$. However, this makes $P \mid Q$ trivial. From Theorem 2.7 we know that for nontrivial $P \mid Q$ the isometry group $G$ is just the edge group generated by the three Schwarz reflections:

$$
\begin{aligned}
& \sigma_{12}=\sigma \\
& \sigma_{13}=r(2 \alpha, 2 \beta) \sigma, \\
& \sigma_{23}=t(2 a, 2 b) r(\pi, \pi) \sigma .
\end{aligned}
$$

From the isometry group

$$
G=\left\langle\sigma_{12}, \sigma_{13}, \sigma_{23}\right\rangle=\langle\sigma, r(2 \alpha, 2 \beta), t(2 a, 2 b) r(\pi, \pi)\rangle,
$$

we wish to extract the lattice group $\Lambda$. Helpful in this enterprise are the vertex group

$$
G_{1}=\langle\sigma, r(2 \alpha, 2 \beta)\rangle,
$$

and its cyclic subgroup

$$
R=\langle r(2 \alpha, 2 \beta)\rangle \text {. }
$$

Sets of translations invariant with respect to $G_{1}$, or stars, play a central role in the construction of $\Lambda$. In what follows we will need two stars:

$$
\begin{aligned}
\Sigma & =\{t(2 a, 2 b)\}_{G_{1}}, \\
\Sigma^{-1} & =r(\pi, \pi) \Sigma r(\pi, \pi)=\{t(-2 a,-2 b)\}_{G_{1}} .
\end{aligned}
$$

The main result is contained in the following lemma: 
Lemma 3.1. Let $G$ be the isometry group of the Riemann surface $[P \mid Q]$ generated by the conformal map of the right triangles specified in (62) and let $R$ be defined by (70), $G_{1}$ by (69), $\Sigma$ and $\Sigma^{-1}$ by (71) and (72). If $r(\pi, \pi) \in R$, then $G$ has lattice group $\Lambda=\langle\Sigma\rangle$ and $G=\Lambda G_{1}$; otherwise, $G$ has lattice group $\Lambda=\left\langle\Sigma \Sigma^{-1}\right\rangle$ and $G=\Lambda G_{1} \cup t(2 a, 2 b) r(\pi, \pi) \Lambda G_{1}$.

Proof. First consider the case $r(\pi, \pi) \in R$; then

$$
G=\langle\sigma, r(2 \alpha, 2 \beta), t(2 a, 2 b)\rangle \text {. }
$$

Now consider the group $H=\Lambda G_{1}$, where $\Lambda=\langle\Sigma\rangle$ is normal in $H$. Clearly, $H \subset G$. Moreover, one easily verifies $g H=H g=H$, where $g$ is any of the three generators of $G$. These two facts together show $G=H ; \Lambda$ is clearly the lattice group of $G$.

Next consider the case $r(\pi, \pi) \notin R$. For the generators of $G$ we must now use (68). Consider the group $\tilde{G}=\tilde{\Lambda} G_{1}$, where $\tilde{\Lambda}=\left\langle\Sigma \Sigma^{-1}\right\rangle$ is normal in $\tilde{G}$. Clearly, $\tilde{G} \subset G$. In contrast to the previous case, we can now only verify that $g \tilde{G} g^{-1}=\tilde{G}$, where $g$ is any of the three generators in (68). Thus $\tilde{G}$ is normal in $G . \tilde{G}$ has index at most two, since multiplication of $\tilde{G}$ by the generators of $G$ produces at most two, possibly distinct, cosets: $\tilde{G}$ and $\tilde{G}^{\prime}=t(2 a, 2 b) r(\pi, \pi) \tilde{G}$. However, if $\tilde{G}^{\prime}=\tilde{G}$, then we would have some $\tilde{\lambda} \in \tilde{\Lambda}$ and some $g_{1} \in G_{1}$ such that $\tilde{\lambda} g_{1}=t(2 a, 2 b) r(\pi, \pi)$, or $g_{1}=\tilde{\lambda}^{-1} t(2 a, 2 b) r(\pi, \pi)$. Since $g_{1}$ fixes the origin, $\tilde{\lambda}^{-1} t(2 a, 2 b)$ must be the trivial translation and $g_{1}=r(\pi, \pi)$. This contradicts our assumption $r(\pi, \pi) \notin R$ and we conclude that $\tilde{G}$ and $\tilde{G}^{\prime}$ are distinct. Let $\Lambda$ be the lattice group of $G$. Clearly, $\tilde{\Lambda} \subset \Lambda$. Now suppose $\lambda \in \Lambda$ but $\lambda \notin \tilde{\Lambda}$. Since then $\lambda \notin \tilde{G}$, we must have $\lambda \in \tilde{G}^{\prime}$, that is, $\lambda=t(2 a, 2 b) r(\pi, \pi) g_{1} \tilde{\lambda}$ for some $g_{1} \in G_{1}$ and $\tilde{\lambda} \in \tilde{\Lambda}$. However, this implies $r(\pi, \pi) g_{1}=t(-2 a,-2 b) \lambda \tilde{\lambda}^{-1}$, a translation, and we arrive at the contradiction $r(\pi, \pi) g_{1}=1$. Thus $\Lambda=\tilde{\Lambda}$.

\subsection{The Discreteness Restriction}

The requirement that $[P \mid Q]$ is discrete places strong constraints on the angles $\alpha$ and $\beta$ of the right triangles $P$ and $Q$.

Lemma 3.2. If $[P \mid Q]$ is discrete, then $\alpha$ and $\beta$ are rational multiples of $\pi$.

Proof. By Lemma 2.9, $[P \mid Q]$ has a discrete lattice, i.e. the orbit $\Lambda \cdot(0,0)$ is discrete in $X \times Y$. For right triangles, Lemma 3.1 gives us $\Lambda=\langle\Sigma\rangle$ if $r(\pi, \pi) \in R, \Lambda=\left\langle\Sigma \Sigma^{-1}\right\rangle$ otherwise. Thus discreteness of $[P \mid Q]$ implies discreteness of the star $\Sigma \cdot(0,0)$ in $X \times Y$. Since $\Sigma=\{t(2 a, 2 b)\}_{G_{1}}=\{t(2 a, 2 b)\}_{R}, \Sigma \cdot(0,0)$ is just the orbit of $t(2 a, 2 b) \cdot(0,0)$ under action of the group $R$ generated by $r(2 \alpha, 2 \beta)$. Clearly, $\Sigma \cdot(0,0)$ lies in a 2-torus $S_{1} \times S_{1}$ embedded in $X \times Y$. Since $\Sigma \cdot(0,0)$ is discrete, there is a disjoint union of neighborhoods, each containing just one element of $\Sigma \cdot(0,0)$. Moreover, since $R$ is an isometry of $X \times Y$ and acts transitively on $\Sigma \cdot(0,0)$, there is a uniform lower bound on the volumes of these neighborhoods. This implies the existence of disjoint neighborhoods in $S_{1} \times S_{1}$, again with a uniform lower bound on their measure. Since $S_{1} \times S_{1}$ has finite measure, this is only possible if $R$ has finite order. 
Notation. Given positive integers $m$ and $n, \operatorname{GCD}(m, n)$ is their greatest common divisor, $\operatorname{LCM}(m, n)$ their least common multiple.

Lemma 3.2 allows us to write $\alpha=\pi(i / k), \beta=\pi(j / l)$, where $i, j, k$ and $l$ are positive integers and $\operatorname{GCD}(i, k)=\mathrm{GCD}(j, l)=1$. Since $R=\langle r(2 \pi(i / k), 2 \pi(j / l))\rangle$, we identify $n=\operatorname{LCM}(k, l)$ as the order of $R$. A more convenient parameterization is given by

$$
\alpha=\pi \frac{p}{n}, \quad \beta=\pi \frac{q}{n},
$$

where $p=i(n / k), q=j(n / l)$ are positive integers with no common factors that are also factors of $n$.

The transformation

$$
t(\sin \alpha, \sin \beta) r\left(-\frac{\pi}{2},-\frac{\pi}{2}\right) t(-\cos \alpha,-\cos \beta) \sigma
$$

has the effect of replacing the angles $\alpha$ and $\beta$ by $\alpha^{\prime}=\pi / 2-\alpha$ and $\beta^{\prime}=\pi / 2-\beta$ in the definition of the triangles $P$ and $Q$. Without loss of generality we may therefore take the smallest of the four angles $\alpha, \alpha^{\prime}, \beta$ and $\beta^{\prime}$, and rename this angle $\alpha$. Transformation (75), as well as an interchange of the spaces $X$ and $Y$, will then give all other cases of triangles $P$ and $Q$ (in their standard position, orientation and scale). From $0<\alpha \leq \alpha^{\prime}$ we obtain

$$
0<p \leq \frac{n}{4}
$$

The inequalities $\alpha \leq \beta$ and $\alpha \leq \beta^{\prime}$, plus the condition to avoid triviality, $\alpha \neq \beta$, then give

$$
p<q \leq \frac{n}{2}-p
$$

Together, inequalities (76) and (77) have no solution unless

$$
n \geq 6 \text {. }
$$

Since the vertex group $G_{1}$ is generated by $R$ and $\sigma$,

$$
\left|G_{1}\right|=2 n \text {. }
$$

We can use transformation (75), which has the effect of interchanging the angles at vertices 1 and 3, to compute the order of $G_{3}$. Let $n^{\prime}, p^{\prime}$ and $q^{\prime}$ be the integers parameterizing the angles at vertex 3 (as in (74)); then

$$
\begin{gathered}
n^{\prime}=\frac{2 n}{\mathrm{GCD}(2 n, n-2 p, n-2 q)}, \\
\frac{p}{n}+\frac{p^{\prime}}{n^{\prime}}=\frac{1}{2} \\
\frac{q}{n}+\frac{q^{\prime}}{n^{\prime}}=\frac{1}{2}
\end{gathered}
$$


and

$$
\left|G_{3}\right|=2 n^{\prime}
$$

Finally, since the group of the regular vertex is generated by two real curves which intersect at right angles,

$$
\left|G_{2}\right|=4
$$

From (68) we find

$$
\psi(G)=\left\langle G_{1}, r(\pi, \pi)\right\rangle=\langle\sigma, R, r(\pi, \pi)\rangle
$$

for the derived point group of $G$. Recognizing $n=|R|$ as the order of an element of the isometry group of the lattice $\Lambda$, we can use the following theorem of Senechal [11] and Hiller [12], and the requirement of discreteness, to bound $n$ from above.

Theorem 3.3 [11], [12]. Let $N(n)$ be the smallest integer such that the group $\operatorname{GL}(N(n), \mathbb{Z})$ has an element of order $n$, then

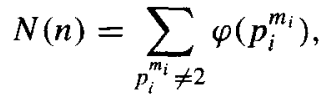

where $p_{1}^{m_{1}} \ldots$ is the prime factorization of $n$ and $\varphi(k)$ is Euler's totient function: the number of positive integers less than and relatively prime to $k$ (note: the prime 2 is included in the sum only if its exponent is greater than 1).

Lemma 3.4. If a Riemann surface $[P \mid Q]$ generated by the conformal map of right triangles $P$ and $Q$ is discrete, then the angles of $P$ and $Q$ are given by (74) and either $n \leq 6$ or $n=8,10$ or 12 .

Proof. Let $G$ be the isometry group of $[P \mid Q]$, let $\psi(G)$ be its derived point group, and let $\Lambda$ be its lattice group. Since conjugation by $g \in \psi(G)$ leaves $\Lambda$ invariant, consider the automorphisms $\Phi_{g}: \Lambda \rightarrow \Lambda$ given by $\Phi_{g}(\lambda)=g \lambda g^{-1}$. If $\Lambda$ has rank $N$, then the homomorphism $\Psi: \psi(G) \rightarrow \operatorname{Aut}(\Lambda)$, where $\Psi(g)=\Phi_{g}$, induces a representation of $\psi(G)$ by integral $N \times N$ matrices of determinant \pm 1 . In fact, $\Psi$ is an isomorphism since for any of the generators $g$ of $\psi(G)$ we can easily find a $\lambda \in \Lambda$ which is not fixed by $\Phi_{g}$ (it suffices to look within the star $\Sigma$ or, if $\left.r(\pi, \pi) \notin R, \Sigma \Sigma^{-1}\right)$. Since $R \subset \psi(G)$ has order $n$, there must be an element of order $n$ in $\operatorname{GL}(N, \mathbb{Z})$. By Theorem 3.3 we must have $N \geq N(n)$. On the other hand, if $[P \mid Q]$ is discrete, then $\Lambda$ must be discrete (as a lattice in $X \times Y$ ) which is possible only if $N \leq 4$. Since $\varphi\left(p^{m}\right)=p^{m-1}(p-1)$, we need only consider the values of $\varphi$ for powers of small primes: $\varphi(4)=2, \varphi(8)=4$, $\varphi(3)=2, \varphi(5)=4$ (all other primes and higher powers yield values greater than 4). From these facts we obtain just the values of $n$ given in the statement of the lemma.

With Lemma 3.4 and inequalities (76) and (77), the set of possible combinations of $n$, $p$ and $q$ is already finite. Several of these combinations can be eliminated by the following lemma which provides a lower bound on $\operatorname{rk}(\Lambda)$ when either $p$ or $q$ is a nontrivial divisor of $n$. 
Lemma 3.5. Let $\Lambda$ be the lattice group of a discrete Riemann surface $[P \mid Q]$ with $P$ and $Q$ defined by parameters $n, p$ and $q$ (74); then if $p>1$ and $p$ divides $n, \operatorname{rk}(\Lambda) \geq$ $\varphi(n / p)+\varphi(n / \operatorname{GCD}(n, q))$ (and the same statement with $p$ and $q$ interchanged).

Proof. Suppose $p$ divides $n$ and $n / p=d>1$. We may assume that $p$ does not divide $q$ since otherwise $n, p$ and $q$ would have $p$ as a common divisor. Let $r=r(2 \alpha, 2 \beta)=$ $r(2 \pi / d, 2 \pi(q / n))$; then $R=\langle r\rangle$ and $R_{p}=\left\langle r^{d}\right\rangle$ is a subgroup of $R$ of order $p$. By looking in $\Sigma$ (or $\Sigma \Sigma^{-1}$ ), we can find a translation $t_{0}=t(u, v) \in \Lambda$ such that $u \neq 0$ and $v \neq 0$. Now consider the two products of translations:

$$
\begin{aligned}
& t_{1}=t\left(u_{1}, v_{1}\right)=\prod_{s \in R_{p}}\left(s t_{0} s^{-1}\right), \\
& t_{2}=t\left(u_{2}, v_{2}\right)=\prod_{k=1}^{d}\left(s_{k} t_{0} s_{k}^{-1}\right),
\end{aligned}
$$

where $s_{k}$ is any element of the coset $r^{k} R_{p}$, and $t_{2}$ depends on the particular choice of coset elements. Evaluating the products we find

$$
\begin{aligned}
u_{1} & =p u \\
v_{1} & =\left(\sum_{m=1}^{p} e^{2 \pi i(q m / p)}\right) v .
\end{aligned}
$$

Equation (88) implies $e^{-2 \pi i(q / p)} v_{1}=v_{1}$, and, since $p$ does not divide $q$, we conclude $v_{1}=0$. Thus $t_{1}=t(p u, 0)$. Similarly, we find

$$
u_{2}=\left(\sum_{k=1}^{d} e^{2 \pi i(k / d)}\right) u=0 .
$$

On the other hand, $v_{2}$ is changed just by making a different choice for one coset element $s_{k}$ (again because $p$ does not divide $q$ ). Thus we can always make a choice such that $t_{2}=t\left(0, v_{2}\right)$, where $v_{2} \neq 0$.

Now $\left\langle\left\{t_{1}\right\}_{R}\right\rangle=\Lambda_{X}$ is a lattice in $X$ isomorphic to the cyclotomic lattice $\mathbb{Z}\left[e^{2 \pi i / d}\right]$, while $\left\langle\left\{t_{2}\right\}_{R}\right\rangle=\Lambda_{Y}$ is a lattice in $Y$ isomorphic to $\mathbb{Z}\left[e^{2 \pi i(q / n)}\right]$. Since $\Lambda \supset \Lambda_{X} \Lambda_{Y}$, $\operatorname{rk}(\Lambda) \geq \operatorname{rk}\left(\Lambda_{X}\right)+\operatorname{rk}\left(\Lambda_{Y}\right)$. The statement of the lemma follows from the well-known formula for the rank of a cyclotomic lattice.

As an example of the application of Lemma 3.5, consider the case $n=8, p=1$ and $q=2$. For these numbers Lemma 3.5 gives $\operatorname{rk}(\Lambda) \geq \varphi(8)+\varphi(4)=6$, and the corresponding Riemann surface would not be discrete. Together, Lemmas 3.4 and 3.5 and the inequalities (76) and (77) limit the set of possibilities for $n, p$ and $q$ to the combinations $(n, p, q)=(6,1,2),(8,1,3),(10,1,3),(10,1,4),(12,1,5)$ and $(12,2,3)$. All other combinations (consistent with the inequalities) correspond to Riemann surfaces whose lattice groups have ranks exceeding 4 and therefore cannot be discrete. We have no general method to settle the discreteness of these remaining candidates and therefore considered them case by case. The procedure was to obtain generators $e_{i}$ of the 
finite sets $\Sigma$, or $\Sigma \Sigma^{-1}$ if $r(\pi, \pi) \notin R$, since these generate the lattice group $\Lambda$. The computations were performed in Mathematica using the function LatticeReduce, which implements the Lenstra-Lenstra-Lovasz (LLL) lattice reduction algorithm. With the exception of the case $(n, p, q)=(10,1,4)$, which was found to have rank 8 , all others proved to have rank 4 and nonvanishing determinant. To these five cases of crystallographic Riemann surfaces, two more should be added which simply correspond to an interchange of the spaces $X$ and $Y$ (obtained by interchanging $p$ and $q$ ). Although the interchangeability of these spaces was assumed in the derivation of inequalities (76) and (77), clearly the sections $\mathcal{A}(x)$ will detect a difference. For example, there is no isometry which relates the surfaces $(10,1,3)$ and $(10,3,1)$ (or their sections). On the other hand, the use of transformation (75) shows that the surface $(10,3,1)$ is isometric with the surface described by $\left(n^{\prime}, p^{\prime}, q^{\prime}\right)=(5,1,2)$ (where now $p^{\prime}<q^{\prime}$ ). However, not all interchanges of $p$ and $q$ produce a new Riemann surface. For example, $(n, p, q)=(8,3,1)$ corresponds, by $(80)$, to $\left(n^{\prime}, p^{\prime}, q^{\prime}\right)=(8,1,3)$ (the values before the interchange).

We summarize this discussion by our main theorem:

Theorem 3.6. Up to linear transformations, there are seven discrete Riemann surfaces $[P \mid Q]$ generated by conformal maps of right triangles $P$ and $Q$. Each of these surfaces is crystallographic; their properties, in particular the integers $n, p$ and $q$ which specify the angles of $P$ and $Q$, are given in Table 1 .

Proof. The properties listed in Table 1 are simple consequences of general results. Since

$$
G / \Lambda \simeq \psi(G)=\langle\sigma, R, r(\pi, \pi)\rangle,
$$

we see that $G / \Lambda$ is isomorphic to an abstract group generated by an element of order 2 , $\tilde{\sigma}$, and an element $\tilde{r}$, which has order $2|R|=2 n$, if $n=|R|$ is odd (and therefore $r(\pi, \pi) \notin R$ ) or $n$ is even and just one of $p$ and $q$ is odd (since then the element of order 2 in $R$ is either $r(\pi, 0)$ or $r(0, \pi)$ ). Otherwise ( $n$ even, $p$ and $q$ both odd), $r(\pi, \pi) \in R$ and $\tilde{r}$ has order $|R|=n$. These generators have the relation $\tilde{\sigma} \tilde{r} \tilde{\sigma}=\tilde{r}^{-1}$ and imply $G / \Lambda \simeq d_{n}$, the dihedral group of order $2 n$, or $G / \Lambda \simeq d_{2 n}$.

Table 1. Properties of the seven discrete Riemann surfaces generated by conformal maps of right triangles. Our notation for $\Lambda$ follows that of [13].

\begin{tabular}{cccccc}
$(n, p, q)$ & $G / \Lambda$ & $\Lambda$ & $g$ & $\rho$ & $\begin{array}{c}\text { Periodic (p) or } \\
\text { quasiperiodic (q) }\end{array}$ \\
\hline$(5,1,2)$ & $d_{10}$ & $A_{4}$ & 2 & $\frac{1}{5} \sqrt{2+2 / \sqrt{5}}$ & $\mathrm{q}$ \\
$(6,1,2)$ & $d_{12}$ & $A_{2} \times A_{2}$ & 2 & $\sqrt{\frac{1}{27}}$ & $\mathrm{p}$ \\
$(8,1,3)$ & $d_{8}$ & $D_{4}$ & 2 & $\sqrt{\frac{1}{8}}$ & $\mathrm{q}$ \\
$(10,1,3)$ & $d_{10}$ & $A_{4}$ & 2 & $\frac{1}{5} \sqrt{2-2 / \sqrt{5}}$ & $\mathrm{q}$ \\
$(12,1,5)$ & $d_{12}$ & $A_{2} \times A_{2}$ & 3 & 1 & $\mathrm{q}$ \\
$(12,2,3)$ & $d_{24}$ & $A_{2} \times Z_{2}$ & 5 & 1 & $\mathrm{p}$ \\
$(12,3,4)$ & $d_{24}$ & $A_{2} \times Z_{2}$ & 5 & $\sqrt{\frac{4}{3}}$ & $\mathrm{p}$ \\
\hline
\end{tabular}


To compute the genus we use the orders of the vertex groups, (79), (81) and (82), in formula (22):

$$
2-2 g=|G / \Lambda|\left(\frac{1}{2 n}+\frac{1}{2 n^{\prime}}-\frac{1}{4}\right) .
$$

The geometry of $\Lambda$ is completely specified by the Gram matrix formed from its generators $e_{k}, k=1, \ldots, 4$ :

$$
\left(M_{k l}\right)=e_{k} \cdot e_{l},
$$

where $\cdot$ is the standard inner product. If we consider each $e_{k}$ as a vector in $\mathbb{R}^{4}$ and form the $4 \times 4$ matrix $E$ whose rows are $e_{k}$, then $M=E E^{\text {tr }}$ and $|\Lambda|=|\operatorname{det} E|=\sqrt{\operatorname{det} M}$. Using the LLL algorithm it was found that the generators of $\Sigma$ and $\Sigma \Sigma^{-1}$ (and hence $\Lambda$ ) could always be written as, respectively,

$$
\begin{aligned}
& e_{k}=2\left(a e^{i 2 k \alpha}, b e^{i 2 k \beta}\right), \\
& e_{k}=2\left(a\left(e^{i 2 \alpha}-1\right) e^{i 2 k \alpha}, b\left(e^{i 2 \beta}-1\right) e^{i 2 k \beta}\right) .
\end{aligned}
$$

To help identify the lattice geometry it was sometimes necessary to define a new basis $E^{\prime}=S E$, where $S \in \operatorname{SL}(4, \mathbb{Z})$. The new Gram matrix is then given by $M^{\prime}=S M S^{\mathrm{tr}}$. Details of this analysis, for the seven combinations of $(n, p, q)$ in Table 1 , are provided in the Appendix.

The only additional data needed in the density formula (48) is the triangle area $|P|=$ $\frac{1}{4} \sin 2 \pi(p / n)$.

The last column of Table 1 identifies which surfaces have doubly periodic sections. In general, since the kernel of the homomorphism $\pi_{X}: \Lambda \rightarrow \pi_{X}(\Lambda)$ is given by the lattice $\Lambda_{Y}=\Lambda \cap Y$,

$$
\operatorname{rk} \Lambda_{Y}=\operatorname{rk} \Lambda-\operatorname{rk} \pi_{X}(\Lambda) .
$$

Surfaces with doubly periodic sections have rk $\Lambda_{Y}=2$, while rk $\Lambda_{Y}=0$ corresponds to completely quasiperiodic sections. These are the only cases that occur, since (from (93) and (94)) $\pi_{X}(\Lambda) \simeq \mathbb{Z}\left[e^{2 \pi i(p / n)}\right]$, the cyclotomic lattice with rank $\varphi(n / \operatorname{GCD}(n, p))$.

\subsection{Piecewise Flat Surfaces and Model Sets}

Suppose a Riemann surface $\mathcal{S}$ is deformed into another surface, $\tilde{\mathcal{S}}$, not necessarily representable locally by graphs of holomorphic functions. As long as the deformation preserves the isometry group and transversality with respect to $Y$, all the crystallographically relevant properties of the point set $\mathcal{A}(x)$ will be maintained in the corresponding deformed point set $\tilde{\mathcal{A}}(x)$. Kalugin's formula (47), for example, makes this invariance explicit for the density.

When a Riemann surface is generated by a triangular graph $P \mid Q$, deformations that preserve isometry and transversality are easily specified by the map defining the fundamental graph, $\tilde{f}: P \rightarrow Q$. We recall that in the Riemann surface, $\tilde{f}$ is holomorphic and extends to a homeomorphism on the closure $\bar{P}$. For the deformed surface $\tilde{\mathcal{S}}$ we continue to use the edge group $G$, defined by the geometry of the triangles $P$ and $Q$, to form the orbit of the fundamental graph, but insist only that $\tilde{f}$ is a homeomorphism. While still preserving isometry and transversality, we even go a step further and modify the topology 
of $\tilde{\mathcal{S}}$ by defining $\tilde{\mathcal{S}}$ as the orbit under $G$ of an open graph. Under these circumstances, when $\tilde{\mathcal{S}}$ is a collection of disconnected components, we are free to relax the condition that $\tilde{f}$ is a homeomorphism. In fact, we are primarily interested in the case when $\tilde{f}$ is a constant map, thereby making each piece of $\tilde{\mathcal{S}}$ flat.

Let $P \mid y$ represent the graph of the constant map, $\tilde{f}(P)=y$. We weigh the merits of various choices of $y \in Y$ as giving optimal "approximations" of the map $P \mid Q$ defined by the conformal map of triangles. By choosing $y=Q_{i}$, a vertex of $Q$, we go the furthest in restoring partial connectedness to $\tilde{\mathcal{S}}$. This is because the action of the vertex group $G_{i}$ on $P \mid Q_{i}$ generates a flat polygon (possibly stellated) composed of $\left|G_{i}\right|$ triangles. Thus $\left|G_{i}\right|$ surface pieces will have been "aligned" by this choice of $y$. With the exception of the $n=5$ and $n=10$ surfaces, $\left|G_{1}\right|=\left|G_{3}\right|=2 n>\left|G_{2}\right|$, suggesting that either one of the singular vertices is a good choice for $y$.

There is another criterion, however, that applies uniformly to all the surfaces and even distinguishes among the two singular vertices. A natural question to ask is: which value of $y \in Q$ "occurs with the highest frequency" in the graph $P \mid Q$ ? To give this question a proper probabilistic interpretation, we suppose that $x$ is sampled uniformly in $P$ and ask for the probability that $\left\|f(x)-y_{0}\right\|<\Delta$, where $f$ is the conformal map of triangles and $\Delta$ is the radius of a small disk about $y_{0} \in Q$. Since $f$ is conformal, this condition (for $\Delta \rightarrow 0$ ) is equivalent to $\left\|x-x_{0}\right\|<\Delta /\left\|f^{\prime}\left(x_{0}\right)\right\|$, where $x_{0}=f^{-1}\left(y_{0}\right)$. Thus the probability of finding $y$ in a neighborhood of $y_{0}$ is maximized by minimizing $\left\|f^{\prime}\left(x_{0}\right)\right\|$. At a singular point $\left\|f^{\prime}\left(x_{0}\right)\right\|$ either vanishes or diverges, and in our case vanishes for $x_{0} \rightarrow$ $Q_{1}$ since we always have $p<q$. We therefore choose $y=Q_{1}$ for all of our surfaces.

The result of flattening the seven Riemann surfaces in Table 1 by this prescription is particularly simple. In all cases $\mathcal{P}=G_{1}\left(P \mid Q_{1}\right)$ is a regular $(n / p)$-gon covered $p$ times (we restore connectedness to $\mathcal{P}$ by including edges incident to vertex 1 ). This means that if each point of $\tilde{\mathcal{A}}(x)$ is counted with multiplicity $p$, then $\tilde{\mathcal{A}}(x)$ and $\mathcal{A}(x)$ have the same density. Using Lemma 3.1, we have

$$
\tilde{\mathcal{S}}=\Lambda \cdot \mathcal{P}, \quad \Lambda=\langle\Sigma\rangle,
$$

if $r(\pi, \pi) \in R$, otherwise,

$$
\tilde{\mathcal{S}}=\Lambda \cup \Lambda t(2 a, 2 b) r(\pi, \pi) \cdot \mathcal{P}, \quad \Lambda=\left\langle\Sigma \Sigma^{-1}\right\rangle
$$

With the exception of the surface $(5,1,2), r(\pi, \pi) \cdot \mathcal{P}=\mathcal{P}$, and we have the simpler description

$$
\tilde{\mathcal{S}}=\langle\Sigma\rangle \mathcal{P},
$$

since

$$
\left\langle\Sigma \Sigma^{-1}\right\rangle \cup\left\langle\Sigma \Sigma^{-1}\right\rangle t(2 a, 2 b)=\langle\Sigma\rangle \text {. }
$$

Figure 1 compares the point sets $\mathcal{A}(x)$ and $\tilde{\mathcal{A}}(x)$, given by the flattening process just described, of the Riemann surface $(5,1,2)$ of Table 1 . Edges have been added to $\tilde{\mathcal{A}}(x)$ to aid in the visualization of three tile shapes: the boat, star, and jester's cap. There is a one-to-one correspondence between the two point sets, with most pairs having quite small separations. In any case, we are guaranteed the separation of corresponding points never exceeds 1 , the diameter of triangle $Q . \tilde{\mathcal{A}}(x)$, the vertex set of a popular tiling model 
[9], is a Delone set. $\mathcal{A}(x)$ fails to be a Delone set because triples of points appear with arbitrarily short separations. That always three points coalesce in this way is a signature of the order of the branch points of $\pi_{X}$ for this surface. $\mathcal{A}(x)$, on the other hand, has a "dynamical" advantage over $\tilde{\mathcal{A}}(x)$. Seen as atoms in a crystal or quasicrystal, the positions $\mathcal{A}(x)$ evolve continuously (in fact analytically) with $x$ (viewed as a parameter), while the atoms in $\tilde{\mathcal{A}}(x)$ experience discontinuous "jumps", and for the most part never move at all. The singular loci of $x \in X$ for the two point sets have different dimensionalities: 0 for $\mathcal{A}(x), 1$ for $\tilde{\mathcal{A}}(x)$; the dynamics of $\mathcal{A}(x)$ is thus more regular also in this sense. To emphasize this point, we note that if $\gamma(t)$ is almost any curve in $X$, then $\mathcal{A}(\gamma(t))$ is a regular homotopy (see Lemma 2.12).

The sets $\tilde{\mathcal{A}}(x)$ are examples of model sets, as defined by Moody [4], and therefore belong to the larger family of Meyer sets. That the set $\tilde{\mathcal{A}}(x)$ shown in Fig. 1 can be organized into a finite set of tile shapes, for example, is a general property of model sets. Model sets obtained by flattening the other six Riemann surfaces of Table 1 are shown in Fig. 3. In the three periodic cases, of course, even $\mathcal{A}(x)$ is a Meyer set and flattening is not necessary if that is our only goal. We flattened these surfaces because the sets $\tilde{\mathcal{A}}(x)$ are then particularly symmetric. In the three quasiperiodic cases the sets $\tilde{\mathcal{A}}(x)$ again organize themselves into tilings that have been discussed in the quasicrystal literature [14]-[16].

It is not true that our restriction on the defining map $f$, that is, one regular vertex of order 2 (Section 3.1), exhausts all crystallographic Riemann surfaces generated by triangular graphs. The simplest counterexample is shown in Fig. 4 (top) and corresponds to a regular vertex of order 3 and angles $\alpha=\pi / 6$ and $\beta=\pi / 2$ at vertex 1 . This Riemann surface has lattice group $\Lambda \simeq A_{2} \times A_{2}$ and, when flattened as in the other examples, yields the section shown in Fig. 4 (bottom).

\section{Acknowledgments}

I thank the Aspen Center for Physics, where a large part of this paper was written. Noam Elkies provided a useful suggestion for the proof of Lemma 2.12.

\section{Appendix}

For each of the entries in Table 1 we give below the corresponding Gram matrix for the generators (either (93) or (94)). From the transformed Gram matrices we see that the lattice geometries are in all cases simple root lattices $\left(A_{n}, D_{n}, Z_{n}\right)$ or direct products. The lattices for $(5,1,2)$ and $(12,3,4)$ are obtained, respectively, from the lattices of $(10,1,3)$ and $(12,2,3)$ by an interchange of the spaces $X$ and $Y$.

$$
M=\left[\begin{array}{rrrr}
6 & 0 & -3 & 0 \\
0 & 6 & 0 & -3 \\
-3 & 0 & 6 & 0 \\
0 & -3 & 0 & 6
\end{array}\right], \quad \Lambda \simeq A_{2} \times A_{2}, \quad|\Lambda|=27
$$



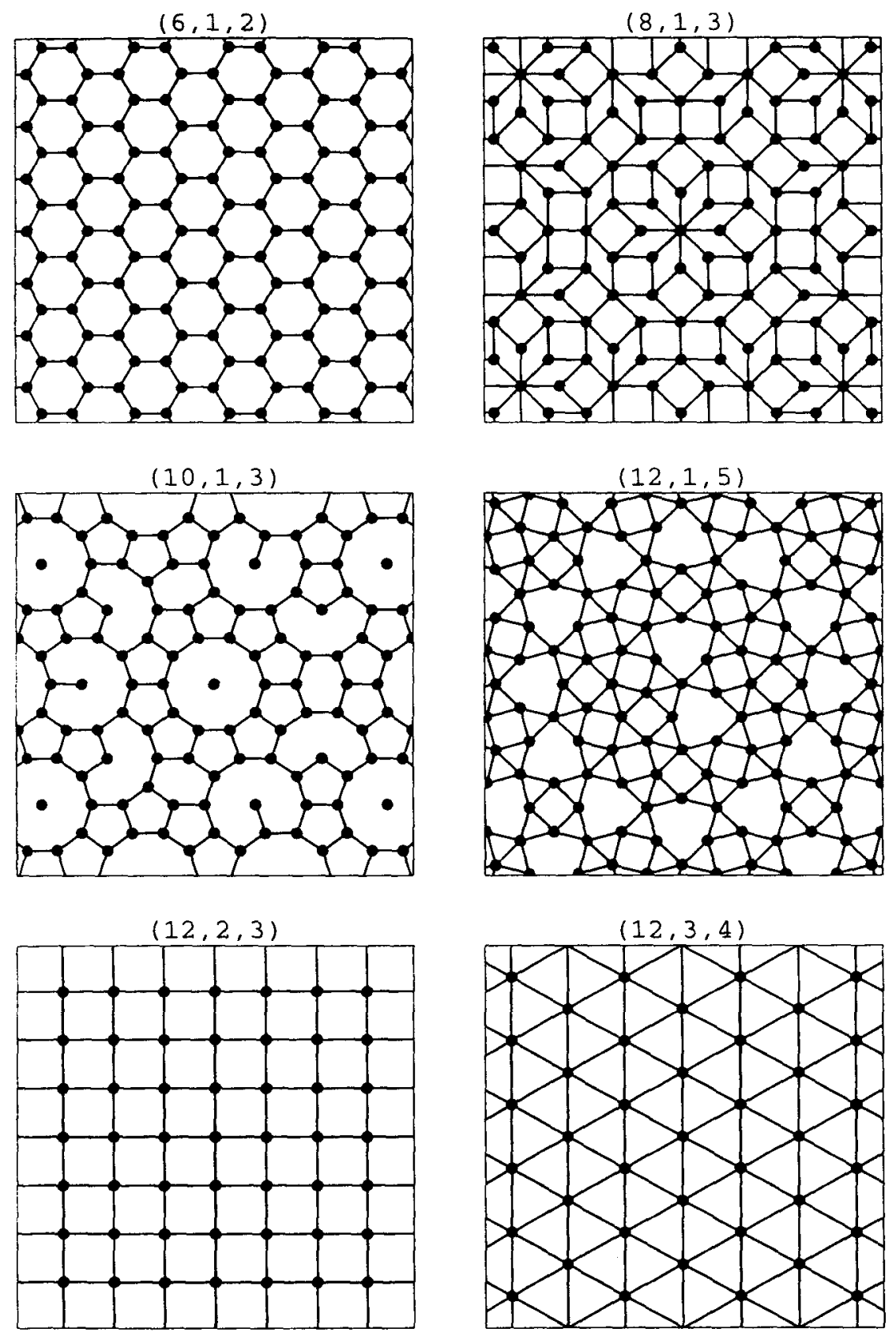

Fig. 3. Point sets given by sections of flattened Riemann surfaces. Lines have been added to help identify tiles. 

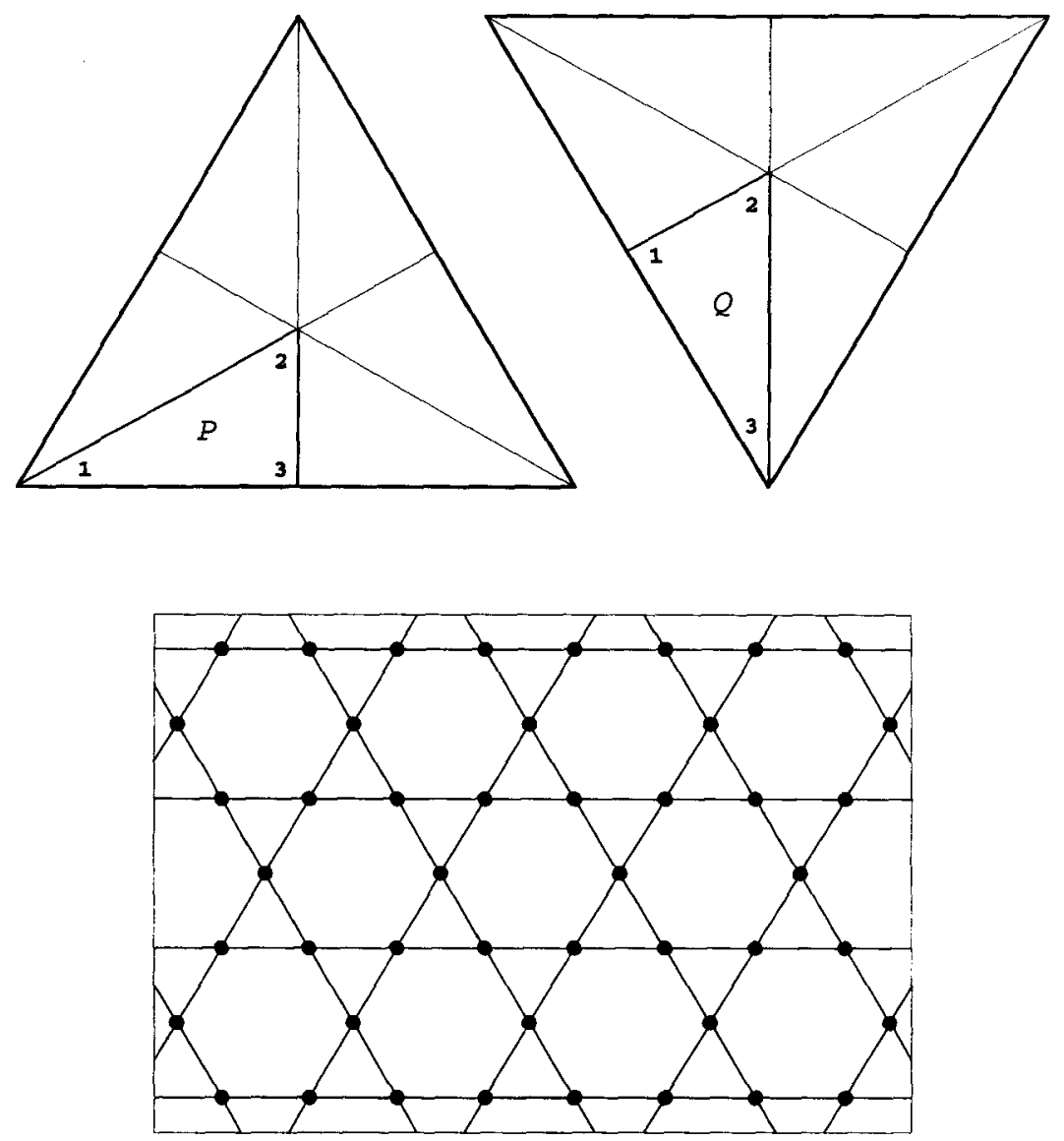

Fig. 4. (top) Example of a map $f: P \rightarrow Q$ with a regular vertex of order 3. (bottom) Section of the corresponding Riemann surface (points) with lines drawn to help visualize a tiling.

$$
\begin{gathered}
M=\left[\begin{array}{rrrr}
4 & 2 & 0 & -2 \\
2 & 4 & 2 & 0 \\
0 & 2 & 4 & 2 \\
-2 & 0 & 2 & 4
\end{array}\right], \quad S=\left[\begin{array}{rrrr}
1 & 0 & 0 & 0 \\
0 & 0 & -1 & 0 \\
0 & -1 & 1 & -1 \\
0 & 0 & 0 & -1
\end{array}\right], \\
S M S^{\text {tr }}=\left[\begin{array}{llll}
4 & 0 & 0 & 2 \\
0 & 4 & 0 & 2 \\
0 & 0 & 4 & 2 \\
2 & 2 & 2 & 4
\end{array}\right], \quad \Lambda \simeq D_{4} \quad|\Lambda|=8 .
\end{gathered}
$$


$(10,1,3)$

$$
M=\frac{1}{2}\left[\begin{array}{rrrr}
10 & 5 & 0 & 0 \\
5 & 10 & 5 & 0 \\
0 & 5 & 10 & 5 \\
0 & 0 & 5 & 10
\end{array}\right], \quad \Lambda \simeq A_{4}, \quad|\Lambda|=\frac{25}{4} \sqrt{5}
$$

$(12,1,5)$

$(12,2,3)$

$$
\begin{array}{cc}
M=\left[\begin{array}{llll}
4 & 3 & 2 & 0 \\
3 & 4 & 3 & 2 \\
2 & 3 & 4 & 3 \\
0 & 2 & 3 & 4
\end{array}\right], \quad S=\left[\begin{array}{rrrr}
1 & -1 & 0 & 0 \\
0 & 0 & 1 & -1 \\
1 & 0 & -1 & 1 \\
0 & 1 & -1 & 0
\end{array}\right], \\
S M S^{\text {tr }}=\left[\begin{array}{llll}
2 & 1 & 0 & 0 \\
1 & 2 & 0 & 0 \\
0 & 0 & 2 & 1 \\
0 & 0 & 1 & 2
\end{array}\right], \quad \Lambda \simeq A_{2} \times A_{2}, \quad|\Lambda|=3 .
\end{array}
$$

$$
\begin{aligned}
& M=\frac{1}{2}\left[\begin{array}{rrrr}
14 & 3 & -1 & -6 \\
3 & 14 & 3 & -1 \\
-1 & 3 & 14 & 3 \\
-6 & -1 & 3 & 14
\end{array}\right], \quad S=\left[\begin{array}{rrrr}
1 & 0 & 1 & 0 \\
0 & 1 & 0 & 1 \\
1 & -1 & 1 & 0 \\
0 & 1 & -1 & 1
\end{array}\right], \\
& S M S^{\mathrm{tr}}=\frac{1}{2}\left[\begin{array}{llll}
6 & 3 & 0 & 0 \\
3 & 6 & 0 & 0 \\
0 & 0 & 8 & 0 \\
0 & 0 & 0 & 8
\end{array}\right], \quad \Lambda \simeq A_{2} \times Z_{2}, \quad|\Lambda|=6 \sqrt{3} .
\end{aligned}
$$

\section{References}

1. D. Shechtman, I. Blech, D. Gratias, and J.W. Cahn, Metallic phase with long-ranged orientational order and no translational symmetry, Phys. Rev. Lett. 53 (1984), 1951-1953.

2. N.D. Mermin, The symmetry of crystals, in The Mathematics of Long-Range Aperiodic Order, R.V. Moody (ed.), Kluwer, Dordrecht, 1997, pp. 377-401.

3. R. Penrose, The role of aesthetics in pure and applied mathematical research, Bull. Inst. Math. Appl. 10 (1974), 266-271.

4. R.V. Moody, Meyer sets and their duals, in The Mathematics of Long-Range Aperiodic Order, R.V. Moody (ed.), Kluwer, Dordrecht, 1997, pp. 403-441.

5. P.A. Kalugin, Density and stoichiometry of icosahedral quasicrystals and related commensurate phases, Europhys. Lett. 9 (1989), 545-550.

6. A. Katz, A short introduction to quasicrystallography, in From Number Theory to Physics, M. Waldschmidt, P. Moussa, J.-M. Luck, and C. Itzykson (eds.), Springer-Verlag, New York, 1992, pp. 496-537.

7. L.S. Levitov, Continuous atomic surfaces, in Quasicrystals: The State of the Art, D.P. DiVincenzo and P.J. Steinhardt (eds.), World Scientific, Singapore, 1991, pp. 239-274.

8. H. Whitney, On singularities of mappings of Euclidean spaces, I, Ann. Math. 62 (1955), 374-410.

9. E. Cockayne and M. Widom, Ternary model of an Al-Cu-Co decagonal quasicrystal, Phys. Rev. Lett. 81 (1998), 598-601. 
10. L.V. Ahlfors, Complex Analysis, 2nd edition, McGraw-Hill, New York, 1966.

11. M. Senechal, Introduction to lattice geometry, in From Number Theory to Physics, M. Waldschmidt, P. Moussa, J.-M. Luck, and C. Itzykson (eds.), Springer-Verlag, New York, 1992, pp. 476-495.

12. H. Hiller, The crystallographic restriction in higher dimensions, Acta Cryst. A 41 (1985), 541-544.

13. J.H. Conway and N.J.A. Sloane, Sphere Packings, Lattices and Groups, Springer-Verlag, New York, 1993.

14. B. Grünbaum and G.C. Shephard, Tilings and Patterns, Freeman, San Francisco, CA, 1987.

15. E. Cockayne, Nonconnected atomic surfaces for quasicrystalline sphere packings, Phys. Rev. B 49 (1994), 5896-5910.

16. F. Gähler, Matching rules for quasicrystals: the composition-decomposition method, J. Non-Cryst. Solids $153 \& 154$ (1993), 160-164.

Received April 21, 1999, and in revised form July 26, 2000. Online publication January 17, 2001. 